\section{LAS ESCUELAS DE ASPLUND: PRIMEROS PROYECTOS,}

\section{RAZONES ENSAYADAS}

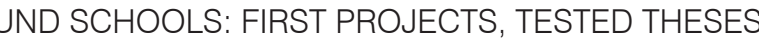

Pablo López-Santana
ASPLUND ANTES DE ASPLUND. ESCUELA KLARA E s ya bien conocido que Asplund comenzó su do transitorio en las artes suecas que propiciabao tipo de cambios. Un par de décadas antes toda una generación de artistas suecos de la talla de Gustaf af Gejerstam o Ernst Josephson que apoyaban los movimientos sindicales y socialdemócratas emergentes en el país se enfrentaron a la Kungliga Akademien för de Fria Konsterna (trad esp. Real Academia de Bellas Artes) reaccionando contra el consenvadurismo académico y fundando como organización independiente el SK (Svensk Konstnärsföbundet: trad esp. Sindicato de Artistas)' Paralelamente en la arquitectura surgió un interés creciente en esta misma dirección desde 1870 que conoció su punto álgido cuando el profesor del KTH (Kungliga Tekniska Högskolan; trad. esp. Real Instituto Tecnológico), Gustaf Carlsson, publico un par de libros a principios del nuevo siglo reivindicando el valor de la arquitectura vernácula², lo cual ahondaba en

as tesis que Isak Clason promulgaba en sus clases ${ }^{3}$. Sin quilosadas en cuestiones arcaicas sin relación alguna con as tendencias del momento en relación a la recuperación de una identidad nacional en todos los ámbitos del arte En ese contexto, una vez que Asplund se gradú en 1909 en $\mathrm{KTH}$ emprendí́ iunto con varios compañeros de promoción unos intentos por introducir cambios en la Konstakademiens Byggnadsskola (trad esp. Escuela de Arte y Construcción) que resultaron fallidos de cara a finalizar sus estudios en el curso de postgrado que además el gobierno sueco exigía desde 1877 para ejercer cargos públicos en el ejercicio profesional. El rechazo a todas las cuestiones planteadas por los estudiantes terminó precipitando que seis de ellos alquilasen un estudio en el edificio de Estocolmo de la calle Klara Västra Kyrkogatan esquina Klarabergsgatan 37 para completar su formación en talleres dirigidos por los profesores que ellos consideraban líderes de toda una nueva generación de arquitectos. A saber: Cart

19d. LNDWALL, Bo. Arisitic Revolution is Nordic Countries. En: Kirk VARNEDOE,. Northerm Light. Realism and Symbolism in Scandinavian Painting, 1880 1910. NY: Brooklyn Museum, 1983, pp. 35-42.

.CARLSSON, Gustaf y MOLIN, Adrian. Svenska All Persona de contacto / corresponding author: Iopezsantanap@gmail.com. Instituto Universitario de Arquitectura y Ciencias de la
Construcción. Universidad de Sevilla. España 


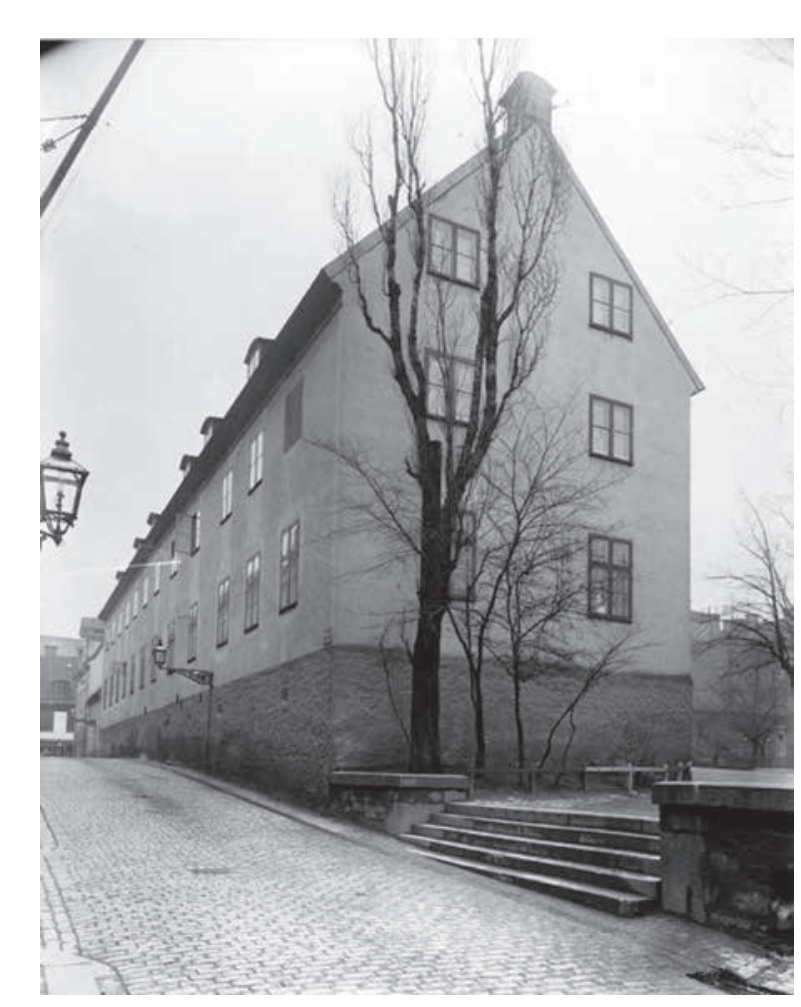

Westman, Ragnar Östberg, Ivar Tengbom y Carl Bergsten De esta forma se fundó -aunque sólo fuera por el curso académico 1910/11- lo que los propios estudiantes denoescogido no son cuestiones baladíes, sino que suponen toda una declaración de intenciones ya que acababan de recrear la Klara Gamla Skola que -desde su fundación en 1649- había sido la principal escuela de estudios primarios de Suecia ubicada precisamente en el edificio que los arquitectos habían elegido para establecer su taller (figura 1). En la década de 1850 estudiaron allí personajes ilustres de la cultura sueca del siglo XIX como el diplomático y poeta Carl Snoilsky, el anatomista Gustaf Retzius o el literato Edvard Bäckström. Pero sin duda, la escuela era conocida por ser donde comenzó su formación, entre 1856 y 1860 August Strindberg, quien terminó por inmortalizarla -junto con el periodista Cläes Lundin- en una crónica de la ciudad editada en $1882^{6}$. Dos años antes de la publicación de Strindberg, la escuela se habia trasladado a Drottningatan

71B al fusionarse con el Stockholms Gymnasium, donde permaneció durante cien años, por lo que la rapsodia a recto de Strindberg y Lundin - plenamente conocida a a Suecia de entonces-cobré especial relevancia para nostálgicos de la época. Así pues, el gesto refundador de os arquitectos se puede considerar como una accín más dirigida a recuperar lo que ellos consideraban un atisbo de autenticidad de su cultura que había sido uno de los motivos principales de su ruptura con la enseñanza académica Esta nueva escuela refería aś a una intimidad profundamente enraizada en la tierra nórdica y de difícil explicación fuera del ámbito cultural de la Suecia del momento.

Pero esta decisión tuvo una serie de consecuencias in-

eludibles en los inicios de Gunnar Asplund. En primer lugar

a nivel intelectual, ya que el haber completado su formación al margen de los planes de estudios oficiales hacía que el estado no le reconociera una titulación al respecto así como los derechos que esta llevaba consigo, como e viaje de estudios. En este sentido Asplund tan sólo pudo contar con una beca tras su graduación en el verano de 1910 para investigar sobre los revestimientos de piedra artificial que se estaban desarrollando en Alemania donde comenzó a forjar su principio de verdad en arquitectura respecto al uso y empleo de materiales, cuestión principal en la época? ${ }^{7}$. Sin embargo no pudo disfrutar de la pensión académica para el viaje de estudios de postgrado, lo que le hizo emprender una travesía por la geografía de su país en 1912 así como un periplo autofinanciado por Italia y el Norte de Africa en el verano siguiente. No obstante, estas travesías alternativas por Suecia y el Sur de Europa se convirtieron en complemento de su primera estancia en el extranjero adquiriendo un caracter más relacionado con motivaciones personales, lo que permite comprender ciertos aspectos de su producción posterior'. Pero más allá de cualquier referencia que influyera en Asplund y se pudiera desprender de estos viajes, lo variado de su recorrido le permitió tomar contacto indistintamente con las

4. Cf. LINN, Björn. Architecture Ancient and Modern. En: Dan CRUICKSHANK (ed.) Erik Gunnar Asplund. Londres: A, 1988, pp. 9-13. Los otros estudiantes fueron Osvald Almqvist, Erik Kalstrand, Sigurd Lewerentz, Josef Ostilihn y Melchior Wernstedt.

5.Vid. LINN, Bjorn. Gunnar Asplund och det Nordiska Luset. En AA.W. Asplund, 1885-1940. Estocolmo: Arkitekturmuseet, 1986, pp. 78 y s.

Strindberg relaciona el término Klara con las monjas de la iglesia del distrito, de las que tomó su nombre la escuela. Cf. STRINDBERG, August y LUNDIN.

7 ASPLUND, Erik Gunnar Nårgra anteckningar om ett modärnt tyskk fasadmaterial. En: Teknisk Tidskrift. Estocolmo: 1911, no7, pp. 100-105.

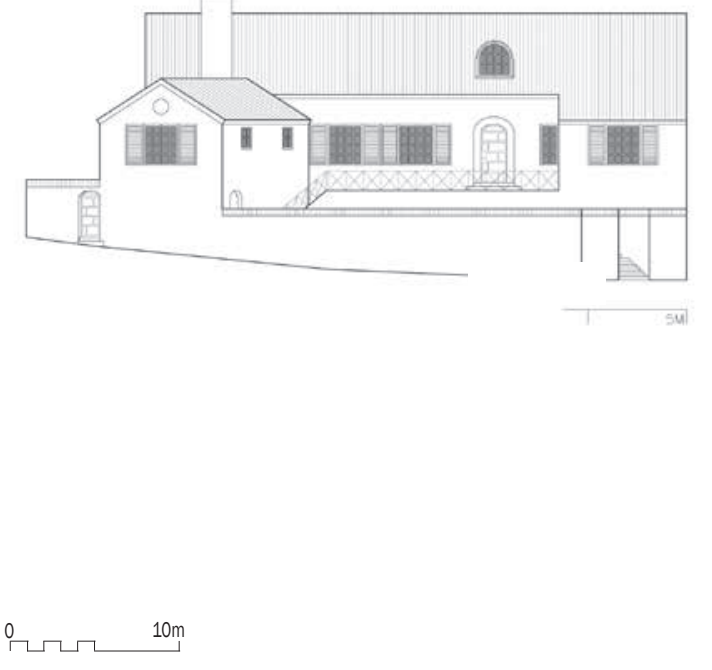

raíces vernáculas y populares de su propia cultura en su viaje de 1912 en sintonia con el espifitu romántico naciona de la Suecia del momento, asi como con las construcciones germánicas yel orden clásico de las distinlas culturas mediterraneas en sus salidas de 1910 y 1913 respectivamente. No obstante cabe senalar que, en cualquier caso las cuestiones que trascendieron de estos viajes nuncallegaron a su trabajo literalmente, sino que fueron trabajadas sulilmente durante toda su carrera.

En segundo lugar existe una consecuencia determ nante en el plano profesional. Y es que el no sometimiento a la última evaluación administrativa en el curso de postgrado oficial le impedia ejercer la función pública, salida muy recurrente para los arquitectos en una Suecia en la que el estado aún resolvia la mayor parte de las construcciones singulares mediante arquitectos propios. Lo cua e obligó a concentrarse durante sus primeros años de carrera en unos pocos encargos de viviendas unifamiliares de allegados. La villas para lvar Asplund (1911), para las familias Rosenberg (1912) y Selander (1913) y para el doctor Ruth (1914) supondran asi pequeños proyec-

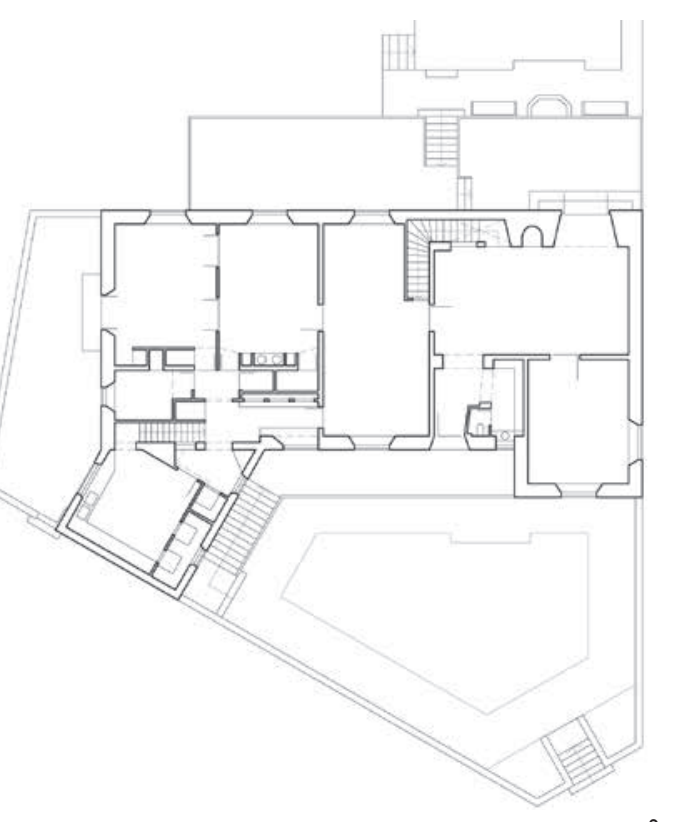

recogidas de sus profesores en la escuela Klara, princarán definitivas en otros proyectos mayores de su obra posterior. Concretamente Wrede en su monograflí llama la atención sobre la inifluencia definitiva que la planta de Ostberg tendra en Asplund, al romper el tradicional cubo compacto mediante inflexiones, asimetrias y el establecimiento de una serie de obliculdades que instauran nuevas tensiones tanto en los espacios interiores como entre los distintos volúmenes construidos en relación a la arquitectura tradicional sueca; asi como del uso de materiales y líneas de acabado - la madera, el ladrillo, los tejados en mansarda .... Todo lo cual conocieron sus estudiantes que compartieron con sus maestros no sólo talleres de trabajo en aquella escuela independiente, sino horas de prácticas colaborando en sus estudios profesionales. De tal modo que en las primeras viviendas unifamiliares de Asplund -principalmente en la villa Rosenberg- se anticipan toda una serie de motivos que se verán desarrollados en la razon de futuros proyectos, concretamente en dos de sus pirmerosi la vila para el banquero Enk Snellman (1917-19) y la escuela Karl Johan (1915-24) (figura 2).

8. Para el viaje a ltalia y Árrica vid. WINTER, Karin. Den Italienska Resan. En: Arkitektur. Estocolmo: 1985, nº, pp. 14-18; HOLMDAHL, Gustav et al. Gunnar Asples Eir de 1912 por Suecia se encuentran recosidas en el archivo AAsplundo del ArkDes. Sin embargo, buena parte de la información se ha perdido ya que Asplund financió su viaje vendiendo sobre la marcha casi todos los dibbjos y pinturas que iba realizando y recuperó el dinero restante vendiendo muchas de sus acua relas a su vuelta.

9. Cf. CALDENBY, Claes. Tiempo, Vida y Trabajo. Una introducción a Asplund. En Claes CALDENBY; Olof HULTIN (eds.) Asplund. Barcelona: GG, 1988, pp. 41-46.

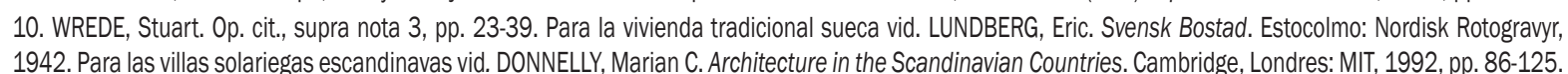


3. Izquierda: Escuela Vägga (Karlshamn, 1912-18) cesos a la villa Rosenbers (izouierda) y a le los ac(derecha). 4. Escuela Vägga (Karlshamn, 1912-18). Planta
baja y reconstrucción de sección de propuesta de

4

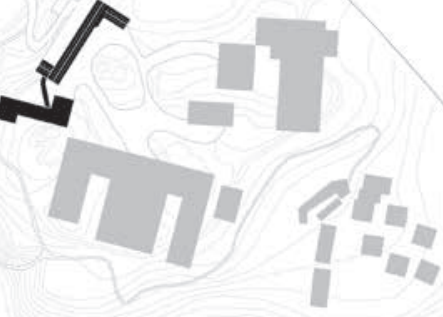

$0 \quad 50 \quad 100 m$

PRIMERAS ESCUELAS. ESCUELA

ÄGGA EN KARLSHAMN

Ahora bien, el cambio de siglo en Suecia fue un contex lo determinante para que Asplund encontrase otro tipo arquitectónico más allá de la vivienda sobre el que volcar sus pintoras tenta ivas. Y para comprender esto es censo al trono de la casa de los Be tomas tas el asXVIV al trono de la casa de los Benadote con Carlos de reafimar su propia dinastí fomenté muy por ende realinar su propla dinastra - fomento muy por enmecenazo de las artes plásticas, as como la for y y én de las poblaciones rurales y clases más desfavoración suecs'1. En este sentido el cuadro Escuel Popular l Archiniélago de Estocolmo (1843) de Jupn Julus Ringdahl es una muestra inequívca de este impulso popular En 1842 el Parlamento sueco aprobó un decreto elativo a la universalización de la educación medionto cual el estado debía proporcionar un sistema educativo obligatorio, público y gratuito, créndose la escuela de educación primaria o folkskolat. No obstante, la crisis cconómica que asoló el pá́s duranto el siglo XIX impidió la construccín de todos los centros que se precisaban para cubrir la nueva demanda, habilitándose cualuvies espacio de dominio municipal existente para estas escuelas, en muchos casos de tamaño reducido por el escaso número de habitantes de algunas zonas recónd

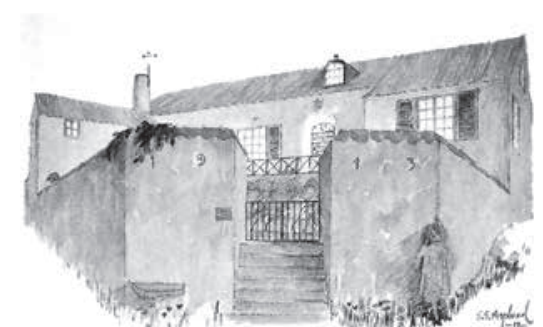

de la geografía sueca y las demandas de los primeros años, tal y como muestra el cuadro de Ringdahl. Sin embargo durante el cambio de siglo con la mejora de la economía y el crecimiento exponencial de las demandas de escolarización, los centros de enseñanza secundaria (Högre Allmänna Läroverket) se dividieron en 1905 en un programa público de seis años (realskola) y un concierto

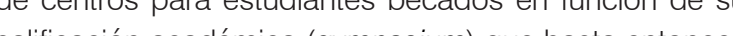

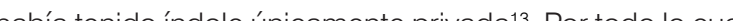
se hizo inpescindible la construcción, amplación ore rma de edicios por todo el pás. El estado no podra asumir este vilum por to trabajo a trav́s de sus técicos municipales lo quepopićc que unimportantenúmedo concursos púlicos a nivel aciont fuer impulsado duante el comienzo del sigloXX. Hecho que signifín una hubiera cursado el programa de postorado oficial, ente (n) Klara y -más concretamente-Gunnar Asplund

De este modo, durante sus primeros años de ejercicio profesional Asplund simultaneó aquellos proyectos de pequeñas viviendas unifamiliares con los diferentes concursos de escuelas a los que se presentón ${ }^{14}$ As'́ las cosas en 1912 mientras proyectaba la casa para berg obtuvo el primer premio para la construcción de un

11. KENT, Neil. The SOul of the North. A Social, Architectural and Cuttural History of the Nordic Countries, 1700-1940. Londres: Reaktion Books, 2000, pp. 272-288. KENT, Neil. A Concise History of Sweden. NY: Cambridge Un. Press, 2008, pp. 154-157 y 168-169.

12. Para un contexto nordicico Vid. DERRY, Thomas K. A History of Scandinavia.. Norway, Sweden, Denmark, Finland and I celand. Londres: Un. Minneapolis Press, 1979, pp. 229 yss.

3. LRÉN, Gösta. Facklärarna i skolans och arbetsmarknadens perspektiv. Estocolmo: Svenska Facklärafförbundet, 1986, passim. 14. De hecho, los honoraraios de esas primeras viviendas y los premios en los concursos de escuelas le permitieron financiar su viaje por talia y Túnez. Cf.

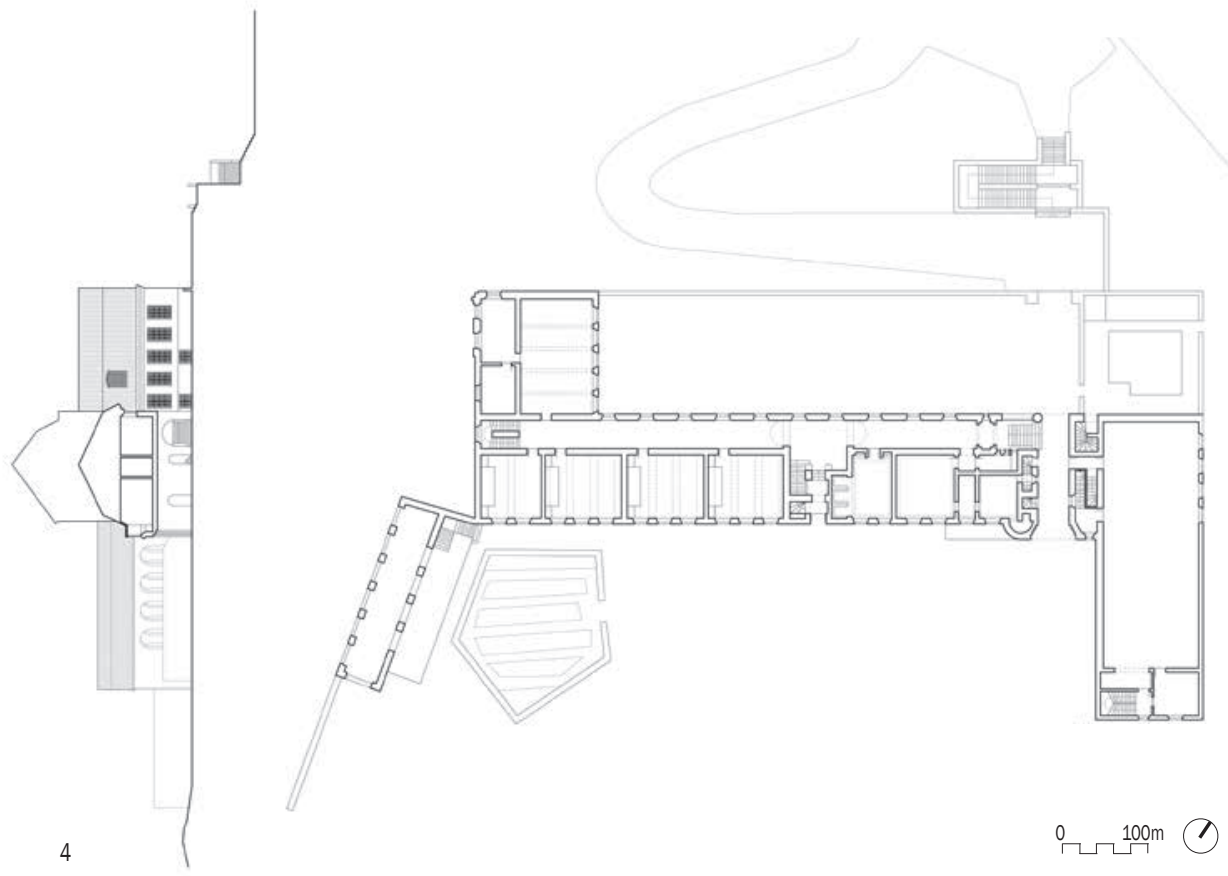

de Karlshamn con un jurado presidido por Isak Clason que señalo que "los planos son excelentes y la gran belleza esructural de la arquitectura está enraizada en sus motivos nacionales"'t5. Aunque la escuela no se consituiría hasta seis años después, este retraso no suscitó cambios sustanciaes en el proyecto en si consistente en un volumen principa de aulas y dirección al que se le adjunta otro perpendicular para gimnasio unidos mediante una galeria-puente. Situado en el punto más alto de un promontorio en la esquina sudeste de la ciudad, Asplund enfrenta su edificio al castill del islote que controlaba la entrada de la ciudad por el Báltco siglos atrás, de modo que reinterpreta el motivo nórdico de las fortalezas y ciudadelas a traves de su disposición en el solar y su privilegiada posición dominante con respecto al mar y al resto de la ciudad'6 (figura 3 ).

Y es que en esta destacada disposición frente al temitorio resuena sin remedio aquel cuadro realizado unos años antes por el pintor romántico nacional Karl Nordström, Fortaleza de Varberg (1893), lo cual resulta especialmente signilicalliv'. La escuela de este modo -vista desde su exterior, desde abajo- remile a la idea de que

a su aislamiento, a su profundidad interior, lo que llama alcuadro de Nordstrom de forma inequivoca cuyo moivo fue visto en la epoca como simbolo identilario de la recuperación de la cullura nórdica'. Asplund asi en la implantación de su escuela resume toda una tradición de ciudadelas y baluartes. En este sentido, Johan Mårtelius ha estudiado las relaciones de la escuela de Karlshamn con distintas obras de Ragnar Ostberg o Isak Gusta Clason', subrayando el hecho de que durante el tiempo que Asplund craboro en el estudio del segundo estuvo al frente del proyecto para el castillo de Adelsnäs en que se dispusieron unos principios similares ${ }^{20}$. Asplun establece una serie de molivos en el acceso gradual a la escuela desde la ciudad a traves del Vaggaparken que serán desarrollados en proyectos futuros como la Biblioteca Nacional de Estocolmo (1924-28) y que complejizaban aquellos que en modo embrionario se presentaban en la villa para los Rosenberg (figura 4).

En este sentido la subida a la colina se desdobla través del parque al disponerse en el arranque de unas escaleras por las que se sube de forma directa-un sen-

15. BERGSTEN, Carl. Priställan om ny realskola i Karlshamn. En: Teknisk Tidskritt. Estocolmo: 1912, n¹0, p. 123. ROSENBERG, Hildring. Karlshamn Historia (1) Karshamn: EG Johansson, 1918-28-49-50-79, pp. 276 y ss.

16. Para castillos y fortalezas vid. DoNNELLY, Marian C. Op. cit., supra nota 10, pp. 73-167.

17. Esta cuestión me la refrendó el propio hijo de Asplund, Ingemar, en una entrevista (Estocolmo, 23/7-2006). Asplund mantenía relación con varios pintores del Romanticismo Nacional, y conocía y admiraba la obra del propio Nordström, amigo personal del arquitecto. El I mededievalismon como respuesta al entorno de

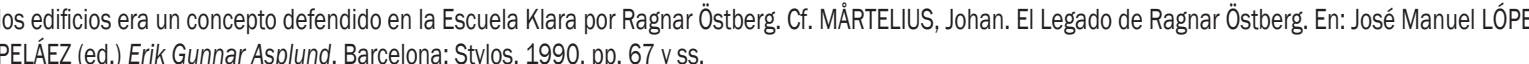

18. FACOS, Michelle. Nationalism and the Nordic Imagination. Swedish Art of the 1890s. Berkeley: Un. California Press, 1998, pp.137-63.

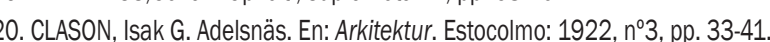




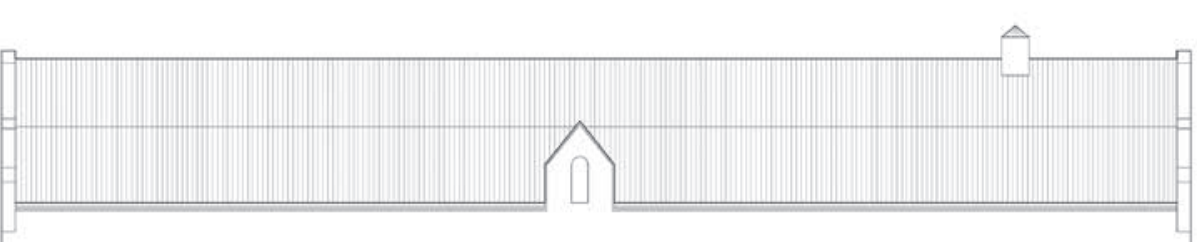

圈

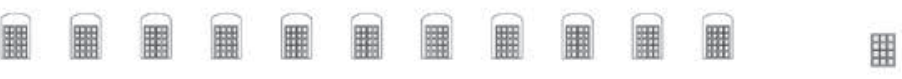
星埇

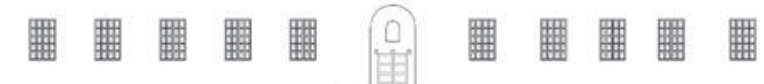

\#曲帮
5. Escuela Väggga (Karrshamn, 1912-18). Fachada Sur. 6. De arriba abajo: Museo Röhsska (C. Westman, 1912-1918). Villa Snellman (Djursholm, 1917-19) 7. Escuela de primaria (Helsingborg, 1909

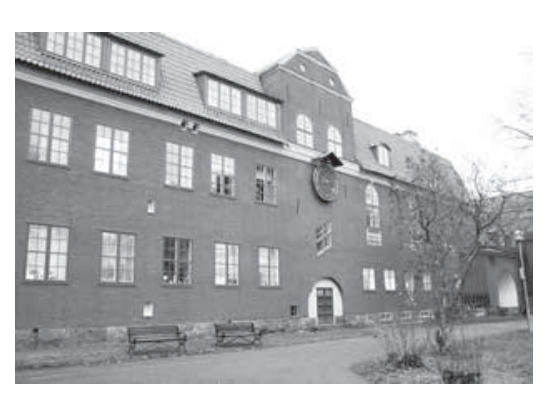

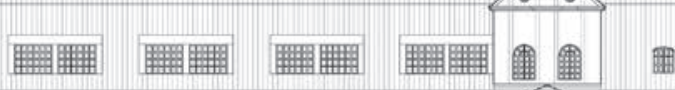

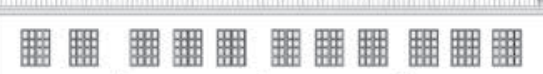

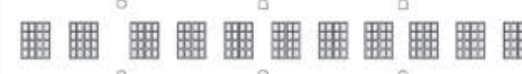
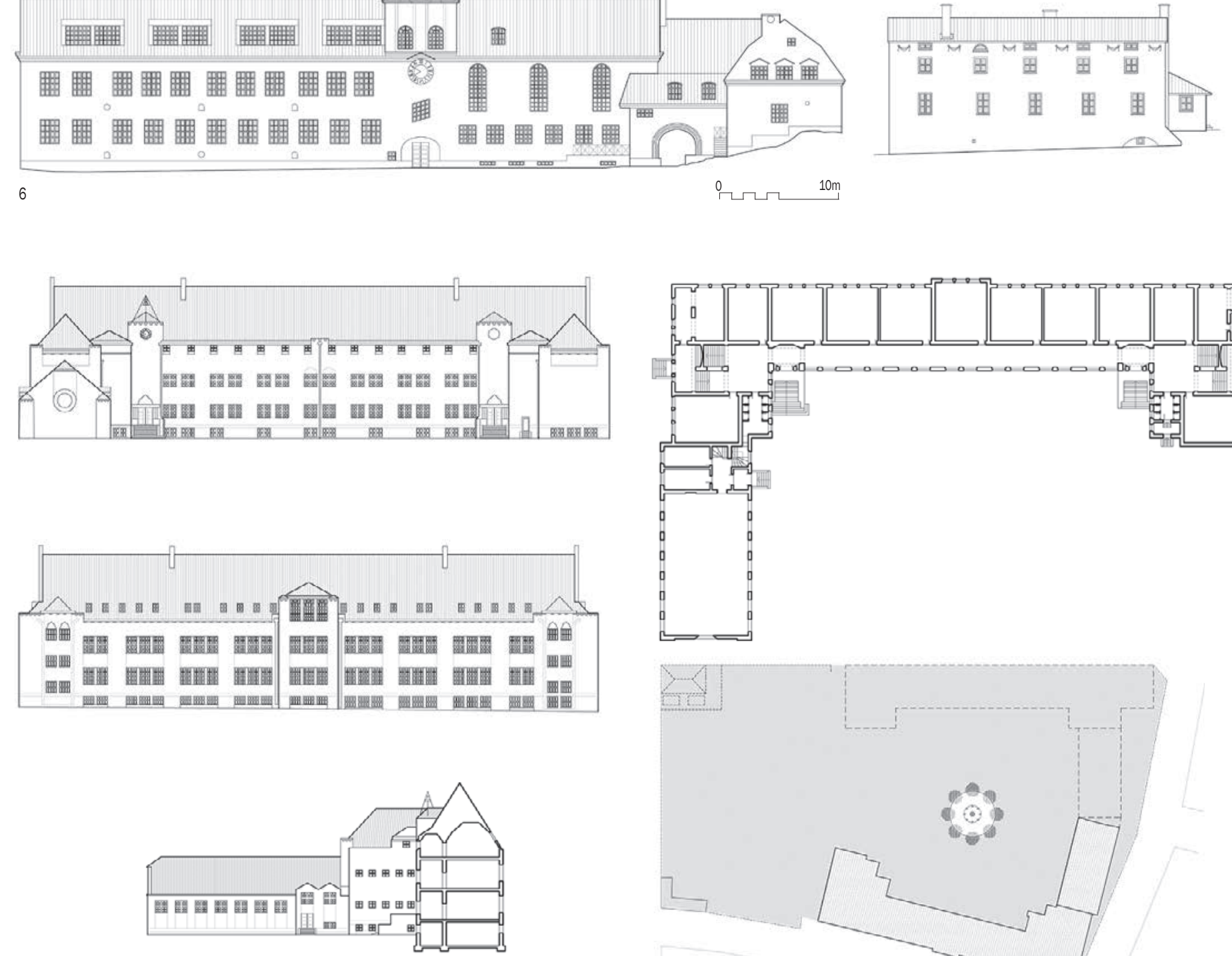

요 $10 \mathrm{~m}$
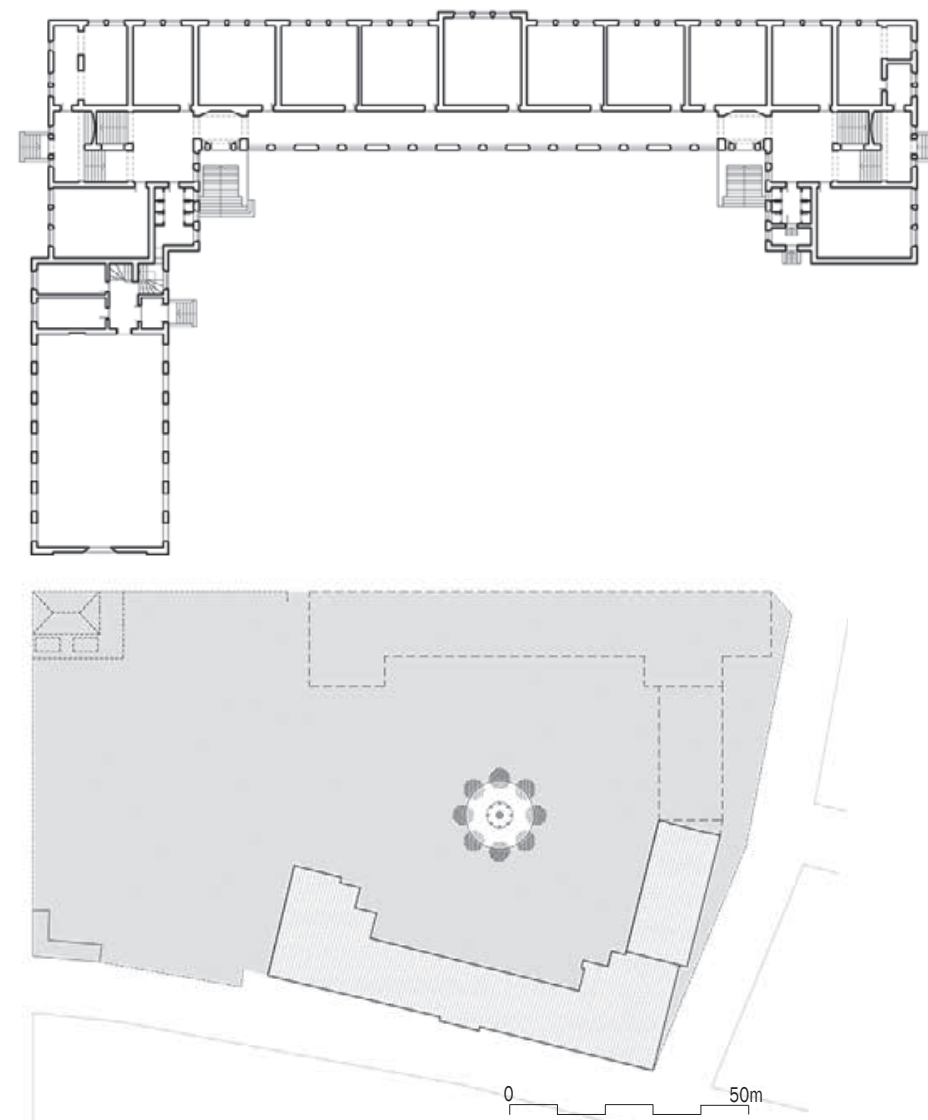

de mínima pendiente del terreno. Ambos recorridos culminan en una especie de vestibulo elevado al aire libre que presenta al edificio en una frontalidad aparente que deja creer una simetria volumetrica a traves de su fachada Norte absolutamente inexistente en su planta. Y este conflicto ya se intuye desde el propio desembarco de ascenso, descentrado con respecto al volumen principa frente a un arco que rompe en planta baja la continuidad del volumen construido a la vez que permite la continuación del recorrido en el espacio por el que se accedía en la propuesta original. Una vez rebasado este, se accede al patio Sur desde donde se aprecia la verdadera disposición asm dica del edificio a través del volumen del gimnasio que queda como cuerpo independiente Este $^{21}$ (figura 5)

Con esta ruptura Asplund se adapta a la topografía del promontorio y termina con el aspecto excesivamente rigido de su fachada Norte. No obstante, ambas fachadas presentan al edificio en un lenguaje romantico nacional en el uso del ladrifilo, tejados inclinados en mansarda y los huecos, tanto en su forma como disposición enrasada con el plano de fachada, lo cual incide en la relación con el cuadro de Nordstrom. En este sentido, además de sus propios proyectos de viviendas, existe una relación inequivoca con la escuela de secundaria de Ostermalm (1910) de Ragnar Ostbergy el Museo Rohsska (1910-14) de Carl Westman?. De hecho, en la obra de este ulltimo se puede apreciar el orden descentrado de los huecos, Ilevada a cabo en la construcción final de Asplund en

21. Para recorridos y transiciones en Gunnar Asplund. Ed. cit., pp. 159-74. 22. Casi con toda seguridad ambas 23. El desplazamiento de las ventanas en Karlishamn se produce por el differente ancho de los pilares de planta baja. Sin embargo en Djursholm el motivo es

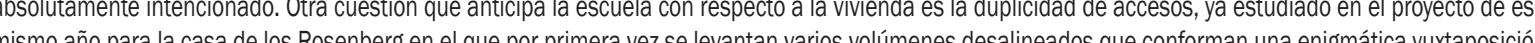
de accesos y distribuciones interiores. Por o otro lado el ladrillo recibió un tratamiento para potenciar su color rojizo (BLUNDELL-JNES. Poter Gunnar asplund

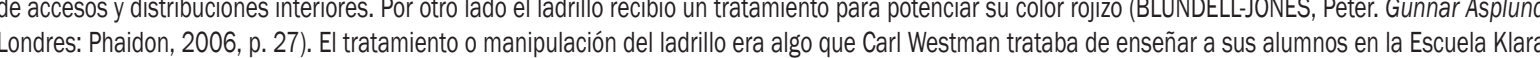
El mismo lo empleó tanto en los Tribunales de Justicia de Estocolmo (1909-15) (WAHLLAN, Lars I S Stokcholms Nya Råahus. En: Teknisk Tidskrift. Estocolmo. 1916, n²2, pp. 13-32 y n³, pp. 33-48) como en el Museo Röhsska de Göteborg (BLANCK, Nils. Röhsska Konstslöjdmuseeti Göteborg. En: Teknisk Tidskritt. Estocolmo: 1917, n2, pp. 18-22. 24. En $1936 \mathrm{el}$ propio Asplund recibió el encargo de ampliar l a escuula. En un lenguaje completamente moderno ubicó su nuevo edificio anejo en ese mismo 


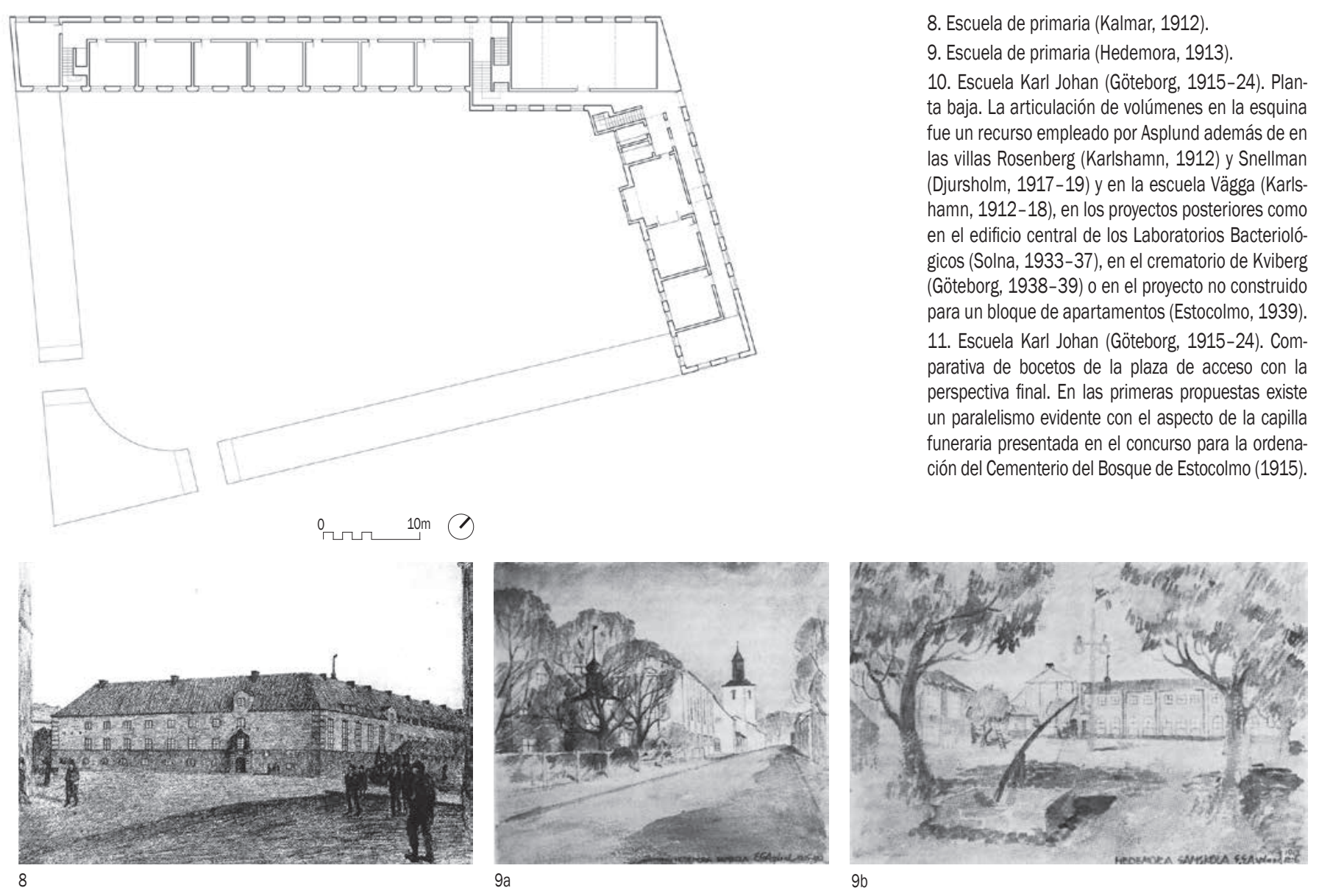

a centralidad en el acceso o el encuentro de volúmenes en esquina, aunque aún no se atreva a romper la ortogonalidac.

En el caso de Kalmar -donde obtuvo el segundo

adquiriendo la severa sobriedad romántico nacional a través del uso del ladrillo visto, tejados en mansarda, buhardillas y la disposición de los huecos.

Al año siguiente Asplund resultó ganador en el concurso para la escuela de primaria de Hedemora mediante unas acuarelas que representaban una perfecta integración en el paisaje urbano en clave romántico nacional quizás si cabe más acusadamente que las anteriores a través de la división de la fachada mediante pilastras y los frontones en el extremo. Sin embargo los clientes consideraron que la obra no era lo suficientemente monumental para el pueblo y no se llegó a construir26 (figura 9).

LA ESCUELA KARL JOHAN

En 1915 Asplund consigue el segundo premio con su propuesta para la ampliación de la escuela Karl Johan en un distrito residencial en construcción al sur de Göteborg. A pesar de no ser elegida en primer lugar, por cuestiones

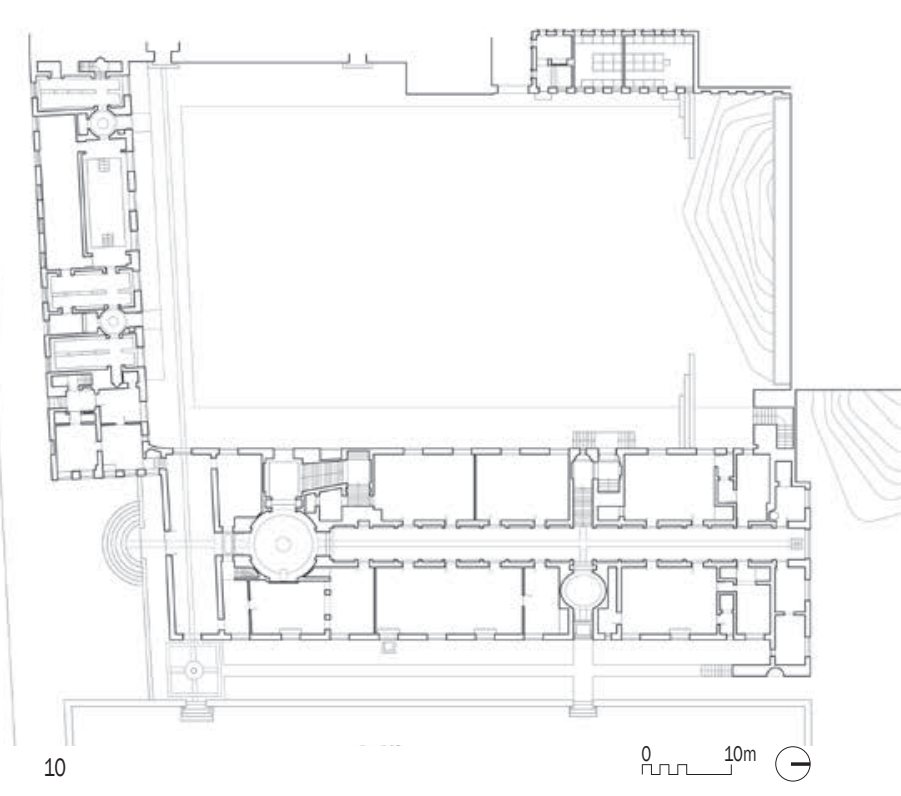

económicas la suya fue la propuesta construida siete años más tarde en un solar que quedaba entre la viea escuela al Este, la plaza Karl Johanstorg al Oeste y el frente de un terreno abrupto al Norte que dejaba su calle trasera diez metros por encima2?. Con estos condicionanes Asplund decide alinear dos volumenes a los frentes libres de la parcela y retomar asi la disposición en $L$ de sus propuestas de la epoca estableciendo una variación urbana de los temas de Karlshamn y Djurlsholm. Es más, en la medida en que el proyecto de Karlshamn supuso octava mayor de la villa para los Rosenberg, esla nueva escuela se puede entender en relación con la vivenda de Snellman como la aten ción de una gran casa pública articula da en su esquina ${ }^{*}$. En este orden, existe una relación de imposibilidad escalar superada por el arquitecto al transtomar una peque na casa familiar de cuatro dormitorios en un colegio de $4000 \mathrm{~m}^{2}$. De modo que se dispone un volumen principa de 5 plantas en dirección N-S con dos crujas de aulas con pasillo central -a diferencia de la crujia y media de Karlshamn- y un pabellon secundario de dos plantas más buhardilia encargado de terminar de cerrar el espacio del patio y conectar volumetricamente la intervención de Asplund con la antigua escuela, en el que se completa

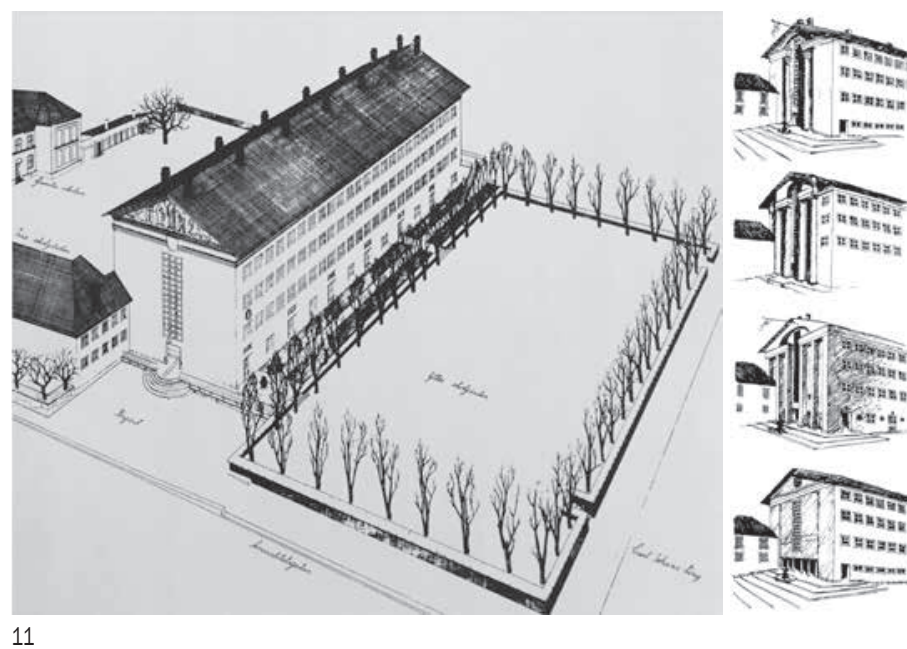

el programa con gimnasio, sala de profesores, talleres vestuarios (figura 10)

Ahora blen, Asplund introduce una variación respecto a sus escuelas anteriores al producir una operación de ruptura en el encuentro en esquina mediante el retranqueo del cuerpo de aulas respecto a la calle Amiralitesgatan. Lo cual no fue una decisión precipitada como 政 esquina (igura 11).

El arquitecto crea de este modo una pequeña plaza con escallinata de acceso que localiza la entrada principa de un edifiro que ha quedado como el juego de dos volúmenes exentos, concentrando todas sus tensiones en un culo llevado a cabo mediante una escalera de $1.25 \mathrm{~m}$. de ancho. Pero ademas esta sutil maniobra permite comprender de forma unitaria y autónoma ese volumen gigante de aulas, de tal modo que se desprende de su figuración una suente de templo que desencadena loda una sucosión de extremos. Y es que las proporciores de su fachada enfiatzan una monumentalidad vertica ematada por aquel timpano de Ivar Johnsson sobre una cornisa jónica que recuerda a la reminiscencia clásica de grupo escultorico de Johan Niklas Byström en el que representaba en mármol al propio Karl Johan como Marte entre

27. lóid, p. 58.

28. Lae escuelay a ha sido estudiada en relación a la arquitectura doméstica, concretamente a las villas de Palladio en KORTE, Martin. Archetypen: Ihre herkun 28.
25. El ganador del concurso fue Osvald Almavist, quién encargóa a Asplund la reseña de su edificio en la que se exponen los proyectos de ambos, así como 26. Los únicos datos que he encontradodo del proyecto han sido en BLUNDEELL-JONES, Peter. Op. Cit., supra notata 23, pp. 29:30. No existen reseñas del concurso

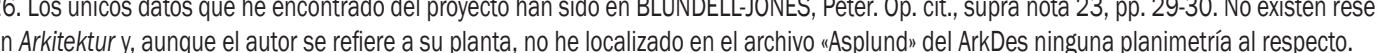




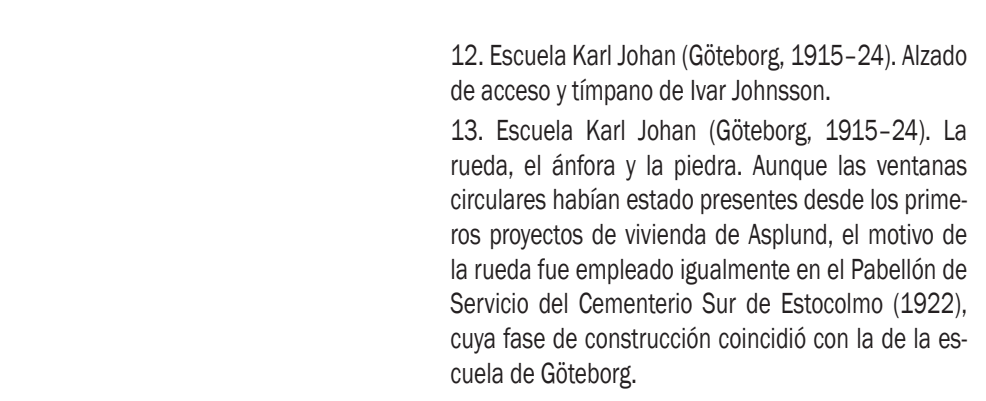

Svea y Nore (1816) (figura 12). De esta forma Asplund ha conseguido refinar aquella entrada lateral que empleara en
sus proyectos anteriores toda vez que se opera una confusión de la frontalidad a través de este frente templario. Y es que a todo templo se accede por su frontón, esto es, por su frente, lo cual parece obvio. Lo que ocurre es que, en contra de lo que sucede en esta escuela cualquier razón funcional dictaría que en la proporcionalidad alargada del cuerpo edificado la fachada principal debeŕa ser cualquiera de las longitudinales, y máxime cuando una de ellas da frente a una plaza pública así como que el acceso debe producirse lo más centrado posible con el objeto de acortar los recorridos. De modo que a través de la articulación de esta pequeña plaza de acceso a la que dispone el frente de su templo, el arquitecto establece un desorden deliberado en la lógica compositiva más elemental sobre la que volveremos a continuación

No obstante, esta imponente fachada guarda otro delicado motivo en su planta: la alineación a la calle es continuada en su plano estableciendo una inflexión en la ortogonalidad supuesta entre el volumen prismático y su cerramiento. A través del deslizamiento de ese plano del prisma se funda un juego de relaciones direccionales que produce un entendimiento global de la intervención en L que su contacto a través de la intersección mínima de los volúmenes parecía estar negando en relación a la escala del conjunto; a la vez que se produce un distanciamiento del concepto ortodoxo del templo clásico en la formalización del prisma truncado de modo que serán los muros interiores los encargados de absorber la irregulaidad contenida en los limites del contexto urbano. Unos Imites donde la roca oculta, aparecida como resultado de las explosiones en los trabajos para aplanar el solar, permanece virgen, intacta, milenaria en su evocación $n^{30}$. Y en el encuentro con esta roca ancestral se revive una especie de experiencia del asombro y angustia de los hombres primitivos a la vista de los espacios abiertos ¿Como adentramos en la intimidad de este contraste entre e edificio recién construido y la roca mantenida por siglos?

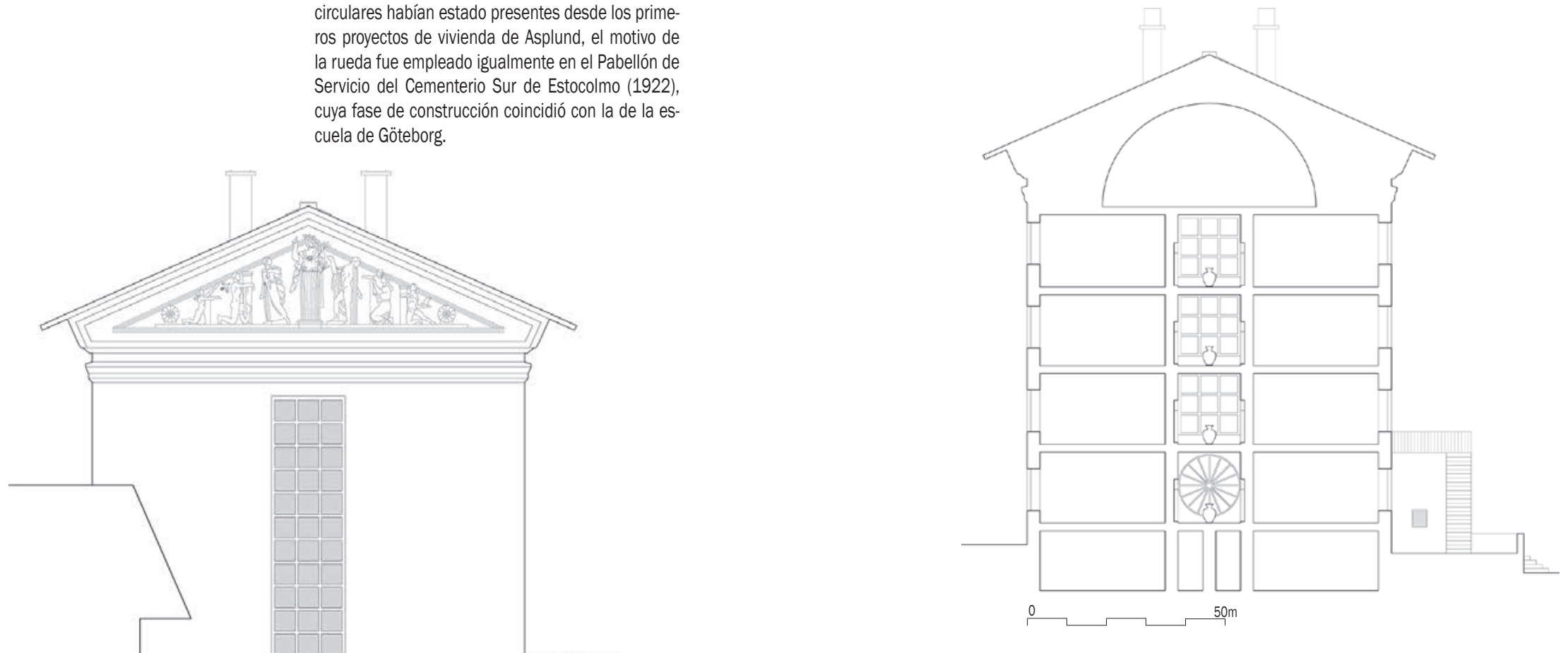

Para ello será suficiente con remitirnos a las palabras ma poed. [... No es la piedra. Es el misterio de la lieSi soderosa y prehumana, que demuestra su fuerza" Si se aplican el sentido psiquico de estas lineas a esa ingen de la roca y la rueda juntas en la variación de porcono Asplund condénsa

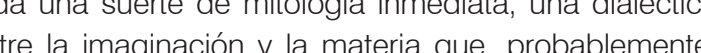
a ha inaginación y la materia que, probablemen, ya hich advertida desde el trabajo de Johnsson, por pre roduce la entrada justamente en er exipal de opuesto a esta piedra en eje con el pasillo principal de distribución de casi 60 metros de largo. Es aqui donde adquiere su sentido el desplazar el acceso del edificio hacia su extremo. Yes que en este corredor el arquitecto convoca una enigmática profundidad en su proporción desmedida sollo interumpida por un juego de tensiones

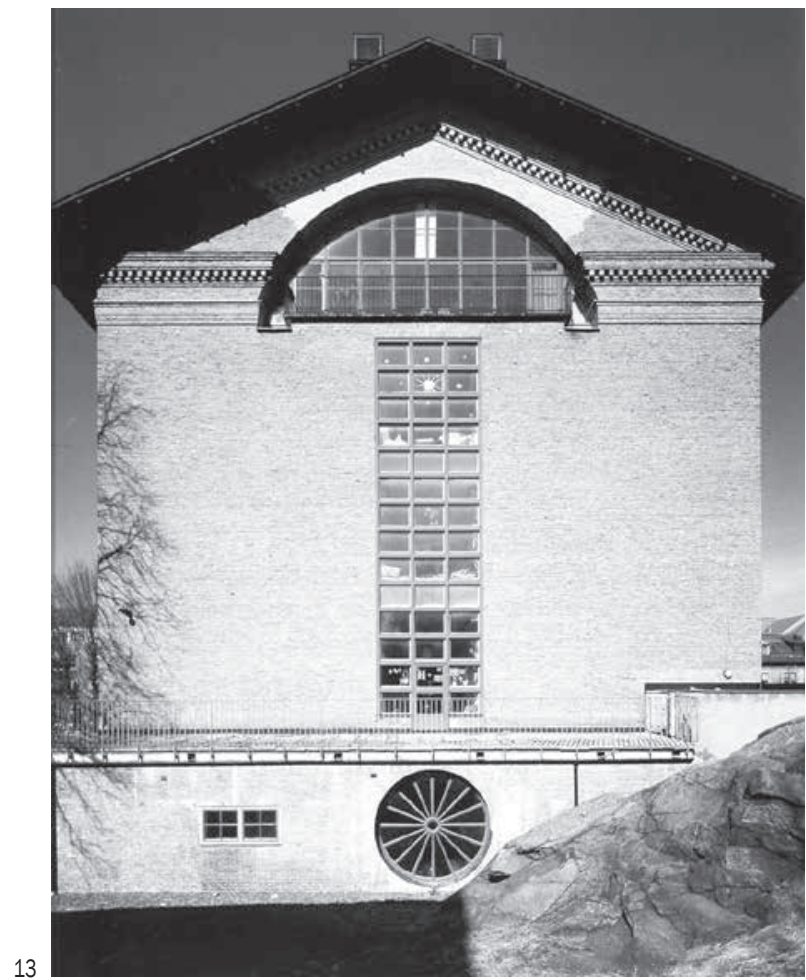

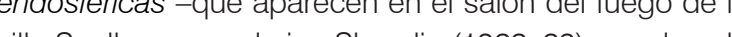
villa Snellman, en el cine Skandia (1922-23) o en la sala de lectura de la Biblioteca de Estocolmo- producidas a introducir una serie de logias curvas en los accesos a los exteriores $\mathrm{E}-\mathrm{O}$ que irrumpen en la concatenación de estancias prismáticas en la planta baja, reservando e untmo molvo cunve en la rueda ventana lias un antora que ilumina alla por el fondo el final del camino, allí donde emerge la roca (figura 13).

Roca, rueda y anfora constituyen así una triada que celebra lo clásico del templo a la vez que convierten a pasillo en un elemento en el que su función de servicropia iNo será esa rueda la apertura neesara pas propia en ćmo sobresale la roca en el exterior?32 Pero. más aún, basta salir al espacio libre que encierran los volúm resistencia que presenta la pared rocosa que enciery el patio y comprender que Asplund - sejendo a la vista aquel espectáculo bizarro- nos ha situado en el interio de una sima. Se nos conduce así a los principios de imaginación material ta roca entonces se convierte en imagen primigenia de amenaza ya que desde su propia inmovilida radica una impresín siempre activa de

31. DH Lawrence cit. pos. BACHELARD, Gaston. La Tierra y los Ensueños de la Voluntad. México DF: FCE, 1994, p. 214 32. Para interpretaciones de la rueda aquívid. LÓPEZ.-PELÁEZZ José Manuel. La Arruitecturara de Gunnar Asplund. Ed. cit., p. 169. 

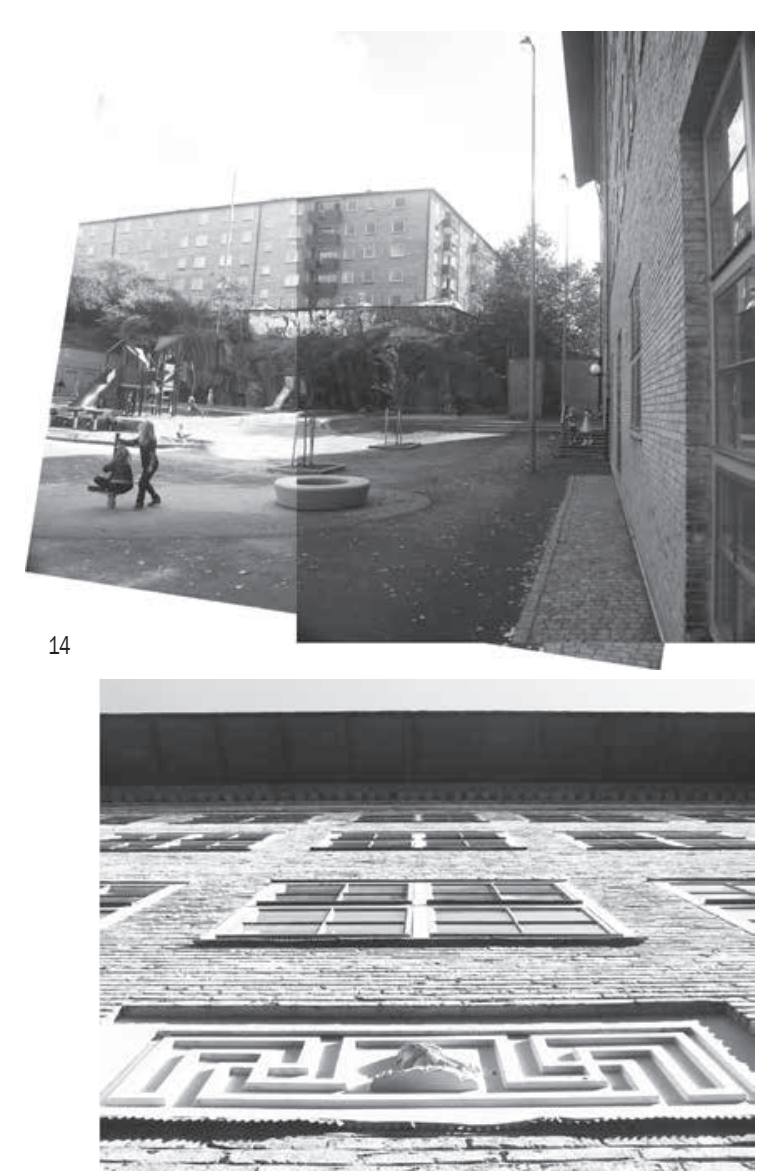

$15 \mathrm{a}$

surgimiento violento, de poderí insondable. Quizás por ello, el consistorio haya decidido vallar aquella parte del perímetro... (figura 14).

Ahora bien, una vez que hemos salido del templo, en sus alzados laterales se advierte un nuevo trastorno de la dialéctica clásica a través del uso indistinto de un enguaje austro-germano con detalles vernáculos para romper el propio orden recurrente. Así, el ladrillo amarillo convoca tanto la obra de Behrens como otorga el mafiz de ciertos edificios escandinavos ${ }^{33}$; mientras que las ventanas cuadradas (en una cierta alusión a Schinkel y Hoffman ${ }^{34}$ ) evitan la verticalidad romántico nacional de la propuesta presentada en 1917, a la vez que se presentan enrasadas con el plano de fachada (recurso empleado recuentemente en la arquitectura nórdica del siglo XVIII). $Y$ en estos laterales se termina de comprobar cómo la cubierta se ofrece como elemento unificador continuo en todo el cuerpo del edificio cerrando a ese templo ralo mediante una solución maquinista, impropia del tipo, al dejar

33. Para el uso del ladrillo en Escandinavia vid. DonNELLY, Marian C. Op. citt, supra nota 10, pp. 18 y ss, 46-83 y 240-47.

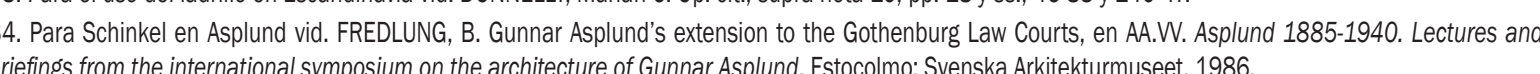

El proyecto de la escuela Karl Johan no deja de ser poston
14. Escuela Kart Johan (Götebors. 1915-24), Patiode recreo. 15. Izquierda de arriba abajo: Escuela Karl Johan
(Göteborg, 1924), propuesta de 1917 y propuesta para el Ayuntamiento de Göteborg (1915). Derecha detalle fachada escuela de Göteborg.

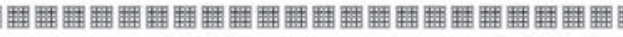

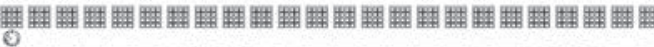

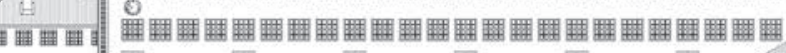

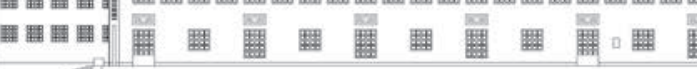

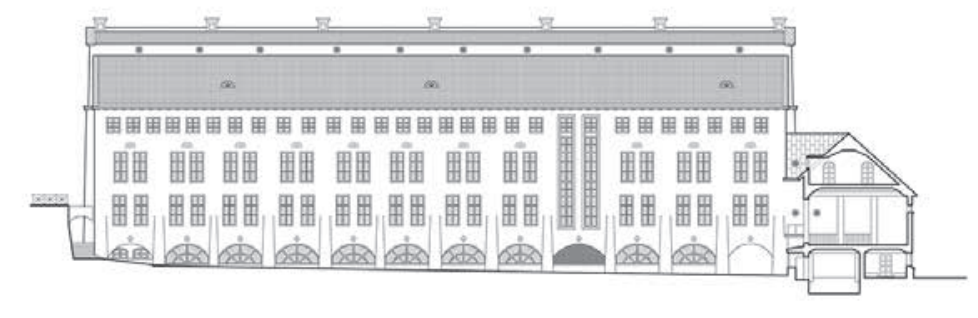
$15 b$

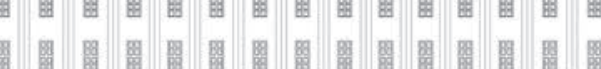

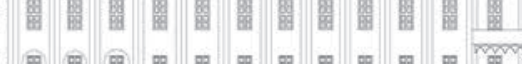

a la vista en su cara inferior su subestructura de pares y entonces un interludio toda vez que supone un estadio de madur en la obra de Asplund respecto a sus primeros thos de profesión consolidando algunos motivos, mientras que simultaneamente trabaja razones de caracter costeriores de su producción. Y esta dualidad imposible como conclusion e intermedio al mismo tiempo se puede (a) el carácter de aquella escuela en entistio de Klara que contribuyo a fundar. Asi las cosas, esculas de Asplund son una medida temprana en toduccín, y estudiarlas exige comprenderlas como a detención de un instante particular en el seno de un ta expresa un método paradigmático en su obra, así como a asimilación por parte del autor de un contexto complejo en la búsqueda permanente de una verdad construida.

\section{Bibliografía citada:}

ASPLUND, Erik Gunnar. Några anteckningar om ett modärnt tyskt fasadmaterial. En: Teknisk Tidskrift. Estocolmo: 1911, nº, pp. 100-105. ASPLUND, Erik Gunnar. Tällan angående ny folkskola i Kalmar. En: Teknisk Tidskrift. Estocolmo: 1913, n³, pp. 29-38. ASPLUND, Erik Gunnar. Carl Johansskolan i Göteborg. En: Bygǵmästaren. Estocolmo: 1925, nº 1, pp. 1-8. AWASHIMA, Yoichi (ed.) E.G. Asplund. Tokio: TOTO, 2005.

BACHELARD, Gaston. La Tierra y los Ensueños de la Voluntad. México DF: FCE, 1994.

BERGSTEN, Carl. Priställan om ny realskola i Karlshamn. En: Teknisk Tidskrift. Estocolmo: 1912, n¹0, pp. 123-126.

BLUNDELL JONES, Peter. Gunnar Asplund. Londres: Phaidon, 2006.

BLANCK, Nils. Röhsska konstslöjdmuseet i Göteborg. En: Teknisk Tidskrift. Estocolmo: 1917, n², pp. 18-22

CALDENBY, Claes. Tiempo, Viday Trabajo. Una introducción a Asplund. En Claes CALDENBY; Olof HULTN, (eds.) Asplund. Barcelona: GG, 1988, pp. 41-46 CARLSSON, Gustaf. Gamla Svenska Allmogehem. Estocolmo: Frtizes, 1912

CARLSSON, Gustaf y MOLIN, Adrian. Svenska Allmogehem. Estocolmo: Fritzes, 1909.

CLASON, Isak G. Adelsnäs. En: Arkitektur. Estocolmo: 1922, nº3, pp. 33-41.

DERRY, Thomas K. A History of Scandinavia. Norway, Sweden, Denmark, Finland and Iceland. Londres: Un. of Minneapolis Press, 1979. DONNELLY, Marian C. Architecture in the Scandinavian Countries. Cambridge, Londres: MIT, 1992.

FACOS, Michelle. Nationalism and the Nordic Imagination. Swedish Art of the 1890s. Londres/Berkeley: Un. of California Press, 1998. FREDLUNG, B. Gunnar Asplund's extension to the Gothenburg Law Courts. En: A..W. Asplund 1885-1940. Lectures and briefings from the interna tional symposium on the architecture of Gunnar Asplund. Estocolmo: Svenska Arkitekturmuseet, 1986.

HOLMDAHL, Gustav et al. Gunnar Asplund, Architect. Plans, Sketches and Photographs. Estocolmo: Byggförlaget, 1986.

KENT, Neil. The Soul of the North. A Social, Architectural and Cultural History of the Nordic Countries, 1700-1940. Londres: Reaktion Books, 2000. KENT, Neil. A Concise History of Sweden. NY: Cambridge Un. Press, 2008.

KORTE, Martin. Archetypen: Irre herkunft und bedeutung bei Erik Gunnar Asplund. Braunschwieg: Technische Universität, 1986.

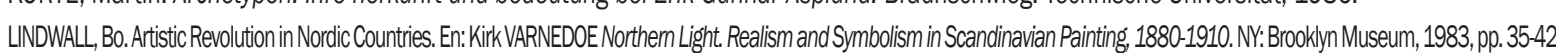
LINN, Bjorn. Architecture Ancient and Modern. En: Dan CRUICKSHANK (ed,) Erik Gunnar Asplund. Londres: A. 1988, po 9-16.

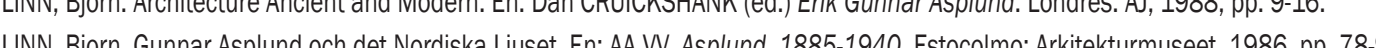

LIRÉN, Gossta. Facklärarma i skolans och Arbetsmarknadens Perspektiv. Estocolmo: Svenska Facklärarföroundundet, 1986.

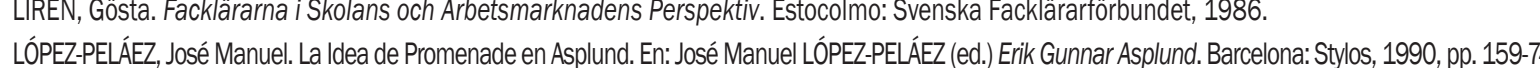
LOPEEZ-PELÁEZ, José Manuel. La Arauitectura de Gunnar Asplunnd. Barcelona: Arquiia, 2002.

LOPEZ-PELAEZ, Jose Manuel. La Arquitectura de Gunnar Asplund. Barcelona. Arquia, 2002. LUNDBERG, Eric. Svensk Bostad. Estocolmo: Nordisk Rotogravary, 1942

LUNDBERG, Eric. Svensk Bostad. Estocolmo: Nordisk Rotograyyr, 1942. MÄRTELIUS, Johan. El Legagado de Ragnnar Östberg. En: José Manuel LOPEZZ-PELÁEZ (ed.) Erik Gunnar Asp

ROSENBERG, Hildring. Karlshamn Historia. 1664-1949. Karlshamn: EG Johansson, 1918-28-49-50-79

STRINDBERG, Augusty L LUNDIN, Cläes. Gamla Stockholm. Estocolmo: Seligmann \& C:is, 1882.

WAHLMAN, Lars I. Stokcholms Nya Rådhus. En: Teknisk Tidskrift. Estocolmo: 1916, n², pp. 13-32 y n³, pp. 33-48.

WINTER, Karin. Den Italienska Resan. En: Arkitektur. Estocolmo: 1985, n6, pp. 14-18.

WREDE, Stuart. La Arquitectura de Erik Gunnar Asplund. Barcelona: Júcar, 1992

Pablo López Santana (Alicante, 1981) es Doctor Arqutecto por EISA (US), Asistente Honorario del Dpto. de Proyectos Arquitectionicos y miembro del Grupo ae Investigacion HUM-632 (US). Sus Investgaciones se centran en el analisis comparado de las distintas disciplinas artísticas en el contaxto nordico-eslavo, fruto de las cuales ha publicado los libros Muerte en el Bosque. Fenomenología una Historia (Athenaica, 2016) sindo este étimo a primera monoerafía editada a nivel mundial de la obra del cineasta succo. 
p.29 ASPLUND BEFORE ASPLUND. KLARA SCHOOL

a sel-known fact that Asplund's architectural training began in 1905, during a transitional period for the Swedish arts open to any type of change. A few decades before, a whole generation of Swedish artists as important as Gusta
af Geeierstam and Ernst Josephson, who supported the emerging union and social democratic movements, had confronted the Kungliga Akademien för de Fria Konsterna (Eng. trans. Royal Academy of Fine Arts) reacting agains scholarly conservatism and founding as independent organization the SK (Svensk Konstnärsfortbundet; Eng. trans. Artist Union)'. A the same time, there had been an increasing interest in the field of architecture pointing in the same direction, since 187 In, hat reached his peak when Gustat Carlsson, professor attherin (Kungliga Tekniska Hogskolan, advocated for the value of vernacular architecture? ${ }^{2}$, thus supporting the thesis that Isak Clason disseminated in his classes'3. This notwithstanding, academic instruction was obsolete, focused on old-fashioned matters completely unrelated to the then current trends regarding the recovery of national identity in all areas of the arts. In this context once Asplund graduated in 1909 trom the KTH, he and some of his classmates tried to introduce some changes a
the Konstakademiens Byggnadsskola, attempt that failed to materialize before finishing their postgradute studies, which the Swedish soverment mandated since 1877 for holding public office and pursuing a profession al activity The rejection of all the issues raised by the students prompted six of them to rent a studio in a building located on Klara Västra Kyrkogatan Street, on the corner of Klarabergsgatan 37 in Stockholm, where they could complete their

p.30 Carl Westman Ragnar Orested by the professors they considered to be the leaders of a new generation of architects academic year $1910 / 11$ - of what the students themselves termed Klara Skola $a^{5}$. Choosing the name of the school and the building for it was not a trivial decision, but instead a statement of intent, since it was a recreation of Klara $G$ amd Skola, which -since its inception in 1649- had been the main Swedish primary school, situated precisely at the same building where the architects had chosen to set up their workshop (figure 1). In the 1850s, distinguished personalities

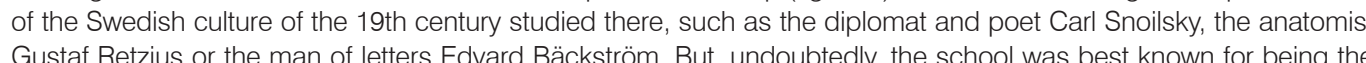
centaf Lundin- would immortalize it in a chronicle of the city printed in $1882^{6}$. Two years before Strindberg's publication, the school was relocated to Drottningatan 71B, after its fusion with the Stockholms Gymnasium, where it remained for a hundred years, reason why the rhapsody by Strindberg and Lundin -very well known in the Sweden of the time acquired special significance for those who back then indulged in nostalgia. In this way, the act of reestablishment authenticity of their culture, one of the main reasons for their breakup with academic instruction. This new schoof referred thus to an intimacy deeply rooted in the Nordic land, difficult to explain outside the context of the Sweden a the time

But this decision had a series of inescapable consequences in the beginnings of Gunnar Asplund. In the first place at an intellectual level, since having finished his studies outside the curriculum of official studies meant not having state
recognition of his degree and not enioying the rights conferred by it such as the study trip In this sense Asplund could only rely on a scholarship atter his graduation the summer of 1910 to do research on artificialal stone cladding that was being developed in Germany, where he began to forge his principle of truth in architecture with respects to the use of materials, key question at the time. . However, he could not obtain the academic pension for the postgraduate study trip, what made him set out on a journey through his country's geography in 1912, as well as a self-financed tour o
Italy and North Africa the following summer Nevertheless, these alternative journeys through Sweden and Souther Europe complemented his first stay abroad and were closer to his personal interests, facilitating the comprehension of certain aspects of his later production. But beyond any other possible reference that could have influenced Asplund after his travel, the diverse nature of the places he visited allowed him to come into contact with the vernacular and

p.31 popular roots of his own culture during his 1912 journey, in harmony with the National Romantic spirit of the Sweden o

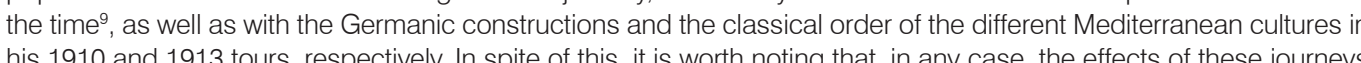
his 1910 and 1913 tours, respectively. In spite of this, it is worth noting that, in any case, the effects of these journeys

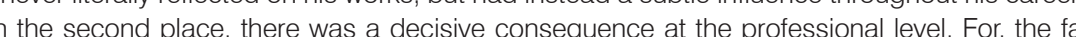
comply with the last requirement of the official postgraduate studies prevented him from working in the public sector

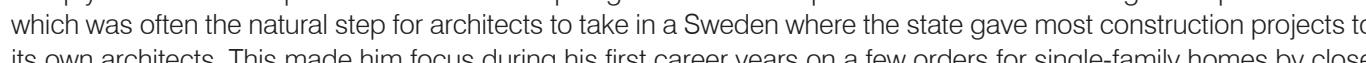
family or triends. The villas for var Asplund (1911), the Rosenberg (1912) and Selander (1913) families, Ruth (1914) would be small-scale proiects in which Asplund showed specific influences acquired from his professors at Klara School, especially from Carl Westman and Ragnar Östberg, that would have a lasting effect in some other bigger projects of his later work. In particular Wrede, in his monograph, draws attention to the definitive influence asymmetries and the establishment of a series of obliquities that create new tensions both in the interior spaces as well as between the different volumes built with respects to traditional Swedish architecture; also, the use of materials and different finishes -wood, brick, mansard roofilo.. All of this was known by the students of that independent school, who not only shared the school's workshops, but also many internship hours working at their professional studios. In this way, in the first few of Asplund's single-amily homes -especially the Rosenberg villa- a series of motifs will be
anticipated, motiffs which will be further developed in his future projects, particularly in two of them: the villa for banker Erik Snellman (1917-19) and Karl Johan School (1915-24) (figure 2). FIRST SCHOOLS. VÄGGA SCHOOL IN KARLSHAMN

That being said, the turn of the century in Sweden provided a decisive context for Asplund to find a different architectura vehicle beyond the home where to put his efforts for his first attempts. And, in order to fully understand this context, with Charles XIV as first monarch, who -with the intention of reinforcing his own dynasty- promoted his country's architecture and the patronizing of the arts way more than his predecessors, as well as the education of the rural population and the lower and disadvantaged Swedish social classes'1. In this sense, the picture Popular School on the the Swedish parliament passed a decree related to the universalization of education that required the state to provide a mandatory educational system, public and free of charge, by which the primary education school or folkskola $a^{12}$ was created. Nevertheless, the economic recession that devastated the country in the 19th century prevented the construction of all the centers needed to meet the new demand, and any free space belonging to the municipality was used for these schools, in many cases of a limited size due to the scarcity of the inhabitants in some remote areas
of the Swedish geography and the demand of the first years, as exemplified in Ringahal's picture. Howeves the of the century brought the improvement of the economy and an exponential increase in the demand for schooling during which secondary education schools (Högre Allmänna Läroverket) in 1905 were divided into centers for a public program of six years (realskola) and a group of centers for scholarship-holder students based on academic merit gymnasium), which until then had only been private institutions $1^{13}$. All of these facts made essential the construction, their municipal technicians, which prompted the creation of an important number of calls for tender at the national level in the course of the $20^{\text {th }}$ century. This fact opened a work possibility to any architect not having gone through an official postgraduate program, among which were those from the Klara School generation and -in particular- Gunnar Asplund.

In this way, during the first year of his professional activity, Asplund worked simultaneously on the projects for small single-family homes and the various calls for tender for schools which he applied for't. In 1912, while working
on the project for the Rosenberg family's villa, he won first prize for the construction of a new secondary school in the same port district of Karlshamn, with a jury presided over by Isak Clason, who commented that athe blueprints are p.33 excellent and the structural beauty of the architecture is rooted in its national motifs, ${ }^{15}$. Although the school was only built six years later, the delay did not imply fundamental changes in the project, which consisted of a main volume of classrooms and administration with an adjoining perpendicular volume for a gym joined by way of a gallery-bridge. controlled the entrance to the city through the Baltic Sea centuries before, so he reinterpreted the Nordic motif of the fortresses and citadels by means of its layout on the site and its privileged position in relation to the sea and the rest of the city' (iigure 3).

And that prominent place in the territory calls to mind that picture painted some years before by National Romantic painter Karl Nordström, Varberg's Fortress (1893), which is of a especial significance $e^{17}$. In this way-seen from the outside, from below-it suggests the idea that its strength lies in the reminiscence to its isolation, to its inner depth, which clearly
elates to Nordstrom's picture, considered in its time an identity symbol of the recovery of Nordic culture's. Thus Asplund, with his school, summarizes an entire tradition of citadels and bastions. Johan Mårtelius has studied the relationships between the school in Karlshamn and different works by Ragnar Östberg or Isak Gustaf Clason'19, highlighting the fact that during the time Asplund collaborated at the latter's studio he was in charge of the project for Adelsnäs Castle, city through Väggaparken that will be developed in future projects like the National Library in Stockholm (1924) which added a level of complexity to the embrynic stage of he project presented for the Rosenber's vilia (figure 4). 
In this sense, the ascension to the hill through the park splits into two, with the arrangement of a winding path at p. the foot of the stanway which provides direct access- hat goes up the hir by taking advantage of the land's minima gradient line. Both ways culminate in a sort of elevated open-air lobby that presents the bullding in a seeming frontality And this conffict can already be sensed at the landing of the ascension, which is not centered in relation to the main And his confifict can already be sensed at the landing of the ascension, which is not centered in relation to the main
volume and facing an arch that breaks with the continuity of the volume built on the ground floor, while at the same time allows for the continuation of the route through the entrance space of the original proposal. Once going through it, one enters the south courtyard, from where the true asymmetric layout of the building becomes visible through the volume for the gym, which constitutes an independent body on the east $\mathrm{t}^{21}$ (figure 5).

By means of this breakup, Asplund adapts to the topography of the hill and does away with the excessive rigidity (of mansard fords picture. In this sense, besides his personal home projects, there exists an unequivocal again relationship to with the Östermalm secondary school (1910) by Ragnar Ostberg and the Röhsska Museum (1910-14) by Carl Westman². In fact, in the latter's work can be seen the off-center order of the recesses, which is not included in the origina
proposal but carried out by Asplund in the final construction of the south facade, anticipating the famos focate From its original ground plan can also be extracted one of the most important details of the proposal, which is also related to the home. an open balcony -which was not built in the end- at a angle to the main volume of the school Beyond the mere rhetorical gesture that may have been the possibility of

After this call for tender, Asplund will apply to two more calls for primary schools in Kalmar (1912) and Hedemora (1913). There is not much information about these proposals. However, both exercises imply interventions of a very
different nature tht National Romantic motits in their appearance. Both exercises can be compared in terms of level to the proposa submitted to a call for tender right after graduating for a primary school in Helsingborg (1909) (figure 7). This one was a sort of minor variation of the one at Karlshamn, with the breaking of the $\mathrm{U}$ ground plan by means of the body of the
$\mathrm{gym}$, which is brought to a side in relation to the rest of the volume in order to break the building's continuity on one of its sides. Still, the elevations show a certain immature rigidity, the faces are not free from the cladding, and the ground plan indulges in a certain symmetry, but it is already possible to see some of the recurrent motifs in the later Asplund

.36 such as the opposition to centrality in the access or the junction of volumes in a corner, atthough he still does not dare break the orthogonality.

In Asplland resumes the tension of confronting different volumes taking advantage of the site's irregularity by arranging
an L-shaped building with two volumes in line with the street, which is at a $100^{\circ}$ angle to the gym as hinge on the corner (figure 8). At both sides he adds an entrance, which goes back to the double access motif, although without the complexity of paths as in the case of Karlshamn. This alignment gave an outward impression of continuity while protecting the south face for the schoolyard. Besldes the significance of tits ground plan with the angled volumes
which could be indicative of its evolution- the building was again presented with the severe austerity of the Nationa

A year later, Asplund won the call for tender for the Hedemora primary school with several watercolors tha way than in the prececedent schools by means of dividing the fational Romantic style, in an even more pronounced end. However, the customers decided that the work was not monumental enough for the town and was never builtz (figure 9)

KARL JOHAN SCHOOL

In 1915 Asplund wins second prize with his proposal for the enlargement of Karl Johan School in a residential distric

p.37 under construction in the south of Göteborg. Despite its second place, and for financial reasons, it would be his Square on the west and opposite a steep plot on the noth which created an unevenness at the back street ten metorg above $^{27}$. Given these conditions, Asplund decides to line up two volumes to the free fronts of the plot, going back to the L-shape of his proposals of the time and establishing an urban variation of the themes tor Karlshamn and Djurlsholm. Moreover, to the extent that the Karlshamn project can be considered as the "big brother" of the Rosenberg's villa,

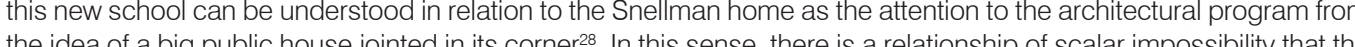
architect overcomes by transforming a small 4 -bedroom family home into a $4000 \mathrm{~m}^{2} \mathrm{~s}$ school. A 5 -story main volume is arranged in north-south direction with two galleries of classrooms at the sides of a central hallway -as opposed to the one and a half galleries of Karlshamn- and a secondary 2-story pavilion plus loft, which closes the space between the yard and provides the volumetric
However, Asplund makes a variation as compared to his previous schools, by creating a breakup in the joint

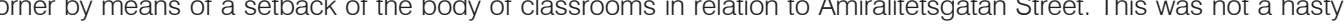
decision, as shown in the series of sketches of the different versions of the corner (figure 11).

nce of a building that remains as the interrelation of two freestanding volumes, uniting all tensions on a ridiculous point carried out by means
of a 1.25 w wide stair Besides, this subtle maneuver allows us to understand the giant volume of classrooms as an autonomous unity, in such a way that its figuration conveys the idea of a temple, which brings about a succession of thoughts. For the dimensions of the facade accentuate its vertical, monumental character, crowned by that tympanum of lvar Johnsson over an lonic cornice that brings to memory the classical reminiscence of the marble sculpture group In this way Asplund succeeded in refining that side entrance used in his earlier projects while creating a confusion of the frontality by means of this temple-like front. And every temple is accessible through its pediment, that is, through p.38 is front, which seems obvious. The fact is that, in opposition to the way this school is built, any functional reasoning would dictate that, in accordance with the elongated proportionality of the built body, the main facade should be any should be as centered as possible in order to shorten the paths. By way of situating this small access squere access of his temple, the architect creates a deliberate disorder of the most elementary compositional logic to which we will hereunder return.

Furthermore, this magnificent facade hides another delicate motif in its ground plan: its alignment with the street continues in its plane, creating an inflection in the assumed orthogonality between the prismatic volume and its
facade. By means of the slip of the prism's plane, a play of directional relationships originates that facade. By means of the slip of the prism's plane, a play of directional relationships originates that generates a global
understanding of the L-shape intervention that its contact through the minimal intersection of the two volumes seemed to be negating in relation to the ensemble's scale; at the same time, it creates a break with the orthodox concept of the classical temple in the formalization of the truncated prism, in such a way that the interior walls will be the ones in charge of absorbing the irregularties contained in the limits of the urban context. Those limits where the hidden

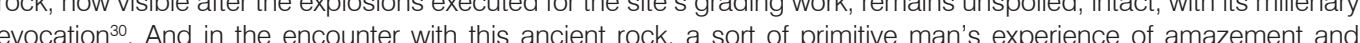
anguish at the sight of open spaces is revived. How could we delve into the intimacy of the contrast between the newly built edifice and the centuries-old rock? It will suffice with bringing to mind the poet's words: $[$ [...] I t is not the stone. It is the mistery of the powerful, pre- $p .39$ human earth, showing its might, $3^{31}$. If we apply the psychic connotation of these lines to the image of the rock and the wheel together in the variation of the archaic temple, we would understand how Asplund condenses a kind of the work by Johnsson, which causes the entrance axis with the main distribution hallway of nearly 60 meters long. It is here where we understand the sense of having moved the building's access to its extreme. For in this corridor the architect brings about an enigmatic depth in its excessive proportions only interrupted by an interplay of endospheric tensions -which are present in the "fire room" at the Snellman villa, in the Skandia theater (1922-23) or at the reading room of the Stockholm Library- created by the
introduction of a series of curved rooms in the accesses to the east-west exteriors that burst into the concatenations of prismatic rooms on the ground floor, resenving the last curved motif on the wheel-window behind an amphora that at the back lluminates the end of the road, there were the rock emerges (ifgure 13).

Rock, wheel and amphora, thus, constitute a triad celebrating the classical reference of the temple, while at the

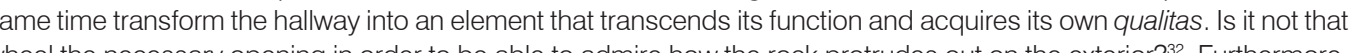
th only takes going out into the free space that the volumes contain to realize a fantasy of strength and resilience that presents the rocky wall enclosing the yard, and to understand that Asplund -by maintaining this bizarre spectacle in sight- brings us right inside a chasm. In this way, we are led to the beginnings of material imagination. The rock then turns into a primitive image of threat, since its very immobility always brings to mind a violent emergence, an $p .40$

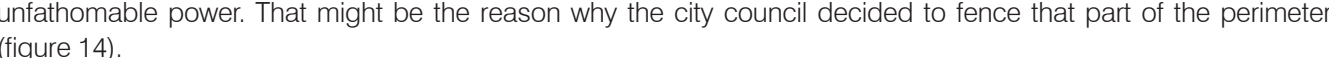

Now then, once outside the temple, we can see a new twist of the classical dialectics on the side facades, by way yellow brick is reminiscent of Behrens' work at the with vernacular touches that break the recurring order. Thus, the have 3 ; while the square windows (an allusion to Schinkel and Hoffman's) avoid the National Romantic verticality of Nordic architecture). And on these side facades can be seen how the cladding is used as a continuous unitying element for all of the building's body, enclosing this rare temple by means of a mechanical solution, untypicical for the ype, leaving on its lower face its substructure of wooden beams (ifigure 15 .

The project for Karl Johan School plays the role of an interlude in Asplinnd's work, with which he also reaches a

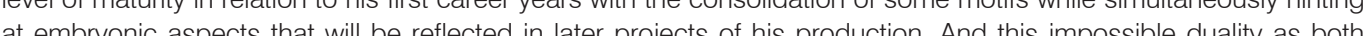


conclusion and transient state can already be understood in the character of that school on the Klara district he contributed to tound. In this state of aftalis, the schools by Asplinnd represent an early stage in his production, and studying them requires understanding them as a brief stop in the course of a work in continual progression. A tested complex context in his continual search for honesty in building.

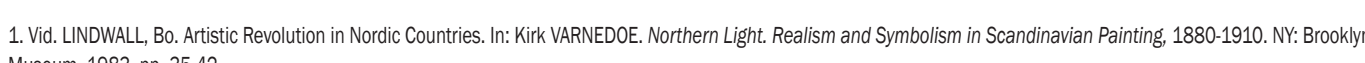

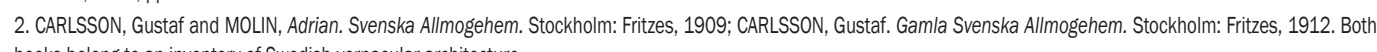
books belong to an inventory of Swedish venaculara architecture.

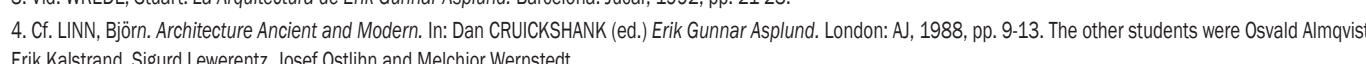

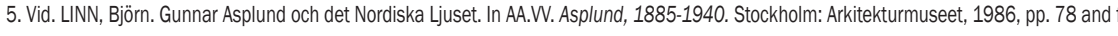

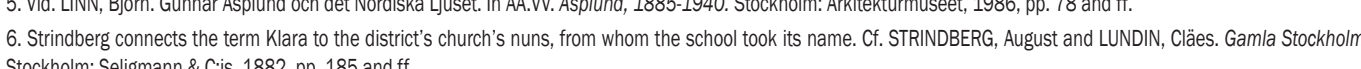

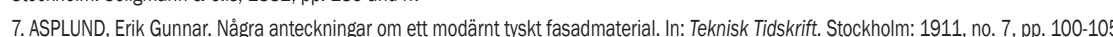

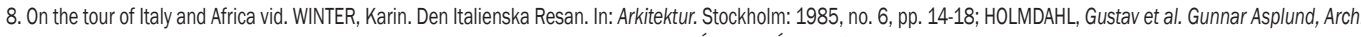

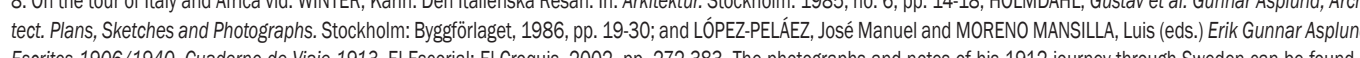

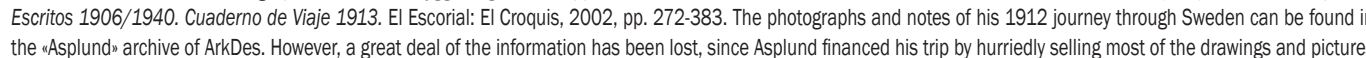
that he made, and goob back the rest of the money by selling many of his watercololis after his retur.

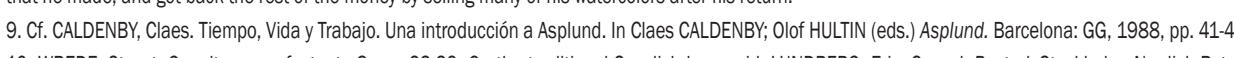

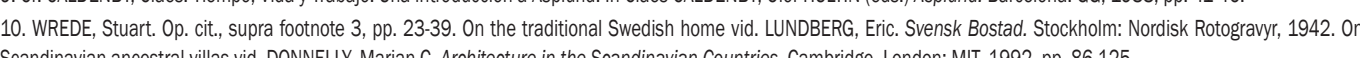

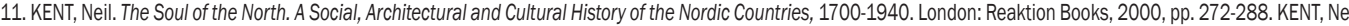

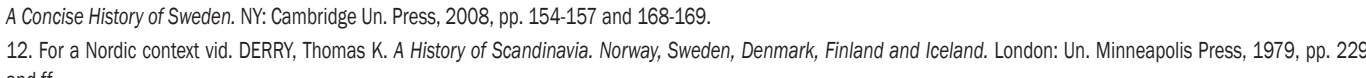

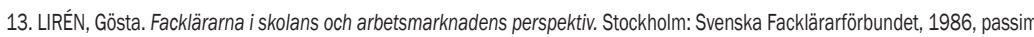

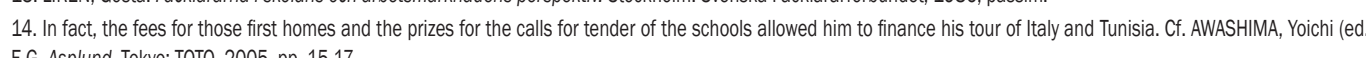

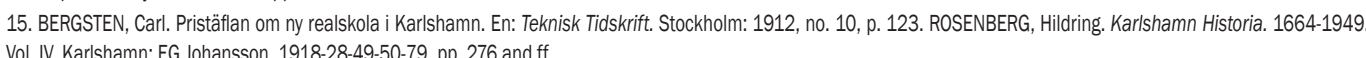

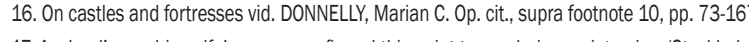

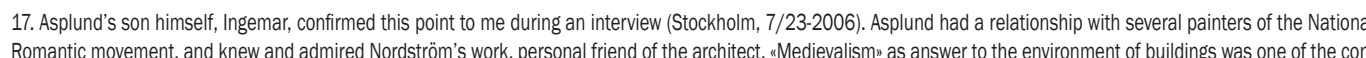

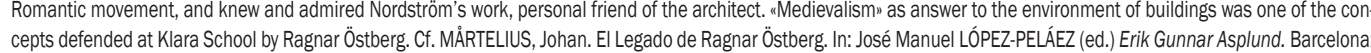
Stylos, 1990, pp. 67 and fit

Berkeley: Un. California Press, 1998, pp.137-63

20.

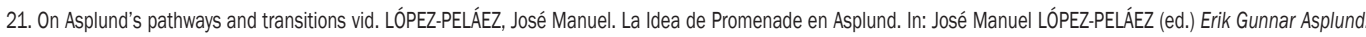
Op. .cit, po. 159.74 .

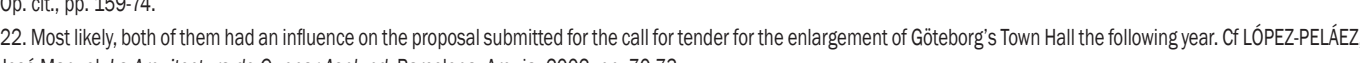

政 question that the school anticipates in relation to the home is the duplicity of accesss, aready studied in the same year's project tor the Rosenberg villa, in which for the firis

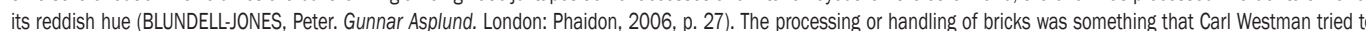

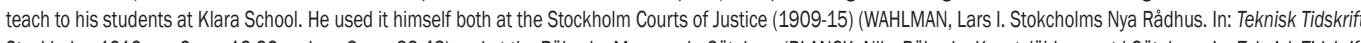

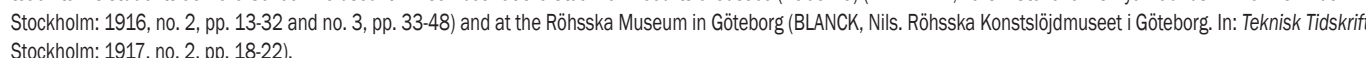

24. In 1936 Asplund himself was commission
reflection of what that balcony could have been

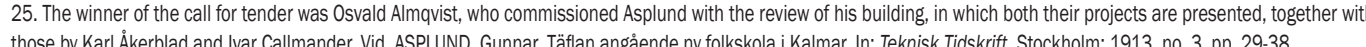

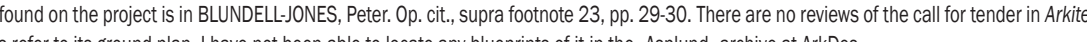
27. bibd, a. .58.

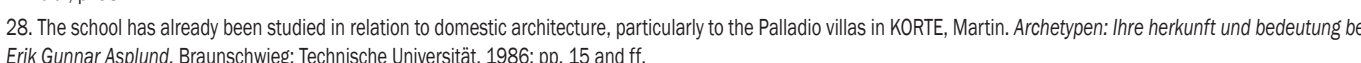

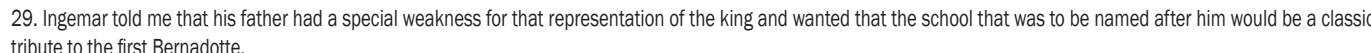

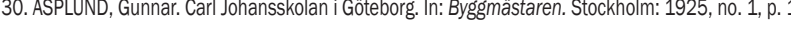

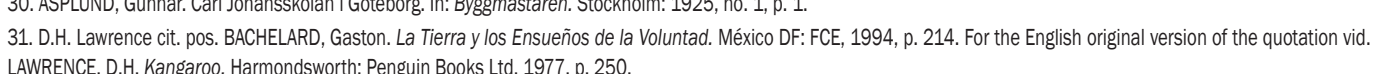

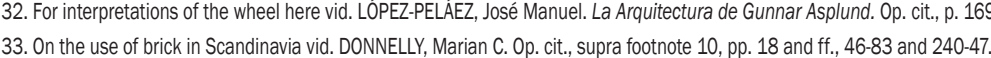

34. On Schinkel in Asplund Vid. FREELLUNG, B. Gunnar Asplund's extension to the Gothenburg Law Courts, en A.W. Asplund 1885-1940. Lectures and briefings from the 


\section{Autor imagen y fuente bibliográfica de procedencia}

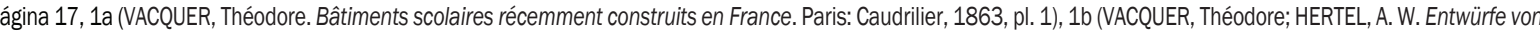

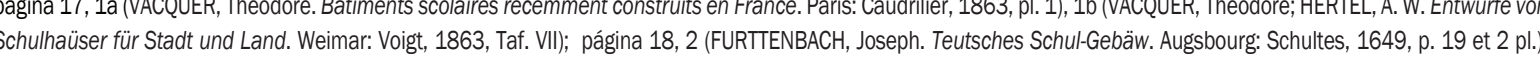

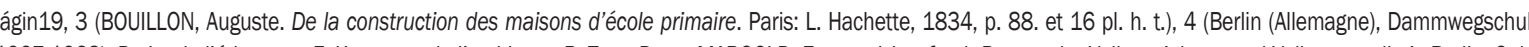

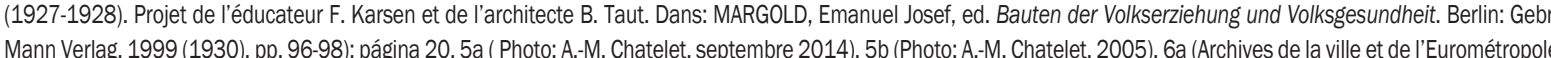
(1) Maquette de la grande percéé; E. Maechling, Musée historique de Strasbourg. Ophoto Musées de Strasbourg, M. Bertola), 7b (GOURLER, BIET, GRILLON et Feu TARDIEU.

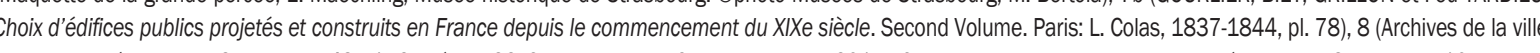

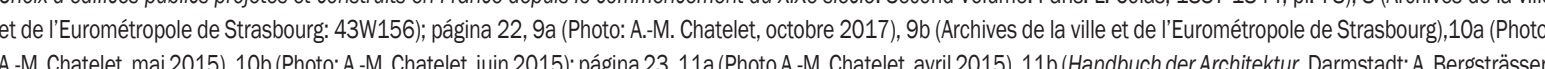

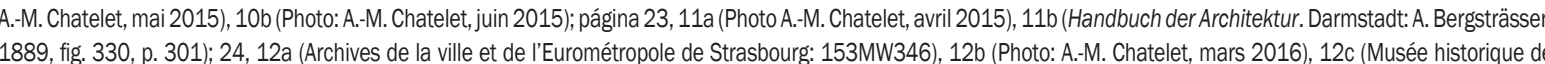

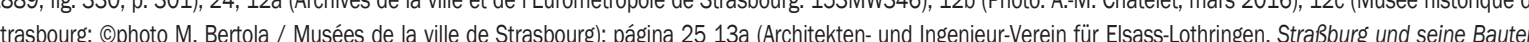
Strassburg: K. J. T. Trübner, 1894, p. 312), 136 (Photo: A.M. Chatelet, décembre 2013), 14a (Photo: A-M. Chatelete, avili 2016), 14 b (Photo: A.M. Chatelet, mai 2015); página

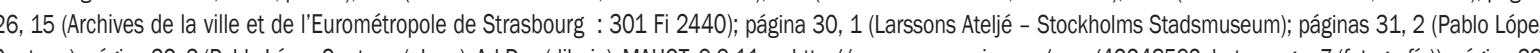

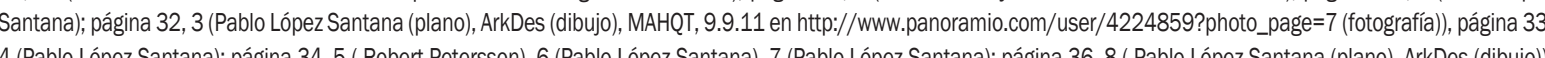

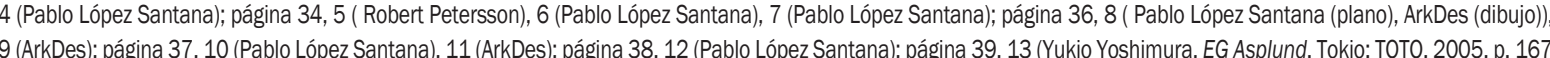

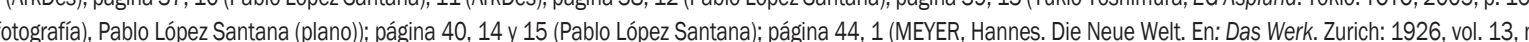
7, p. 218), 2 y 3 (KIEREN, Martin. Hannes Meyer. Dokumente zur Frühzeit Architektur - und Gestatungsversuche 1919 - 1927. Heiden: Niggl, 1990, p. 18; página 47, 4

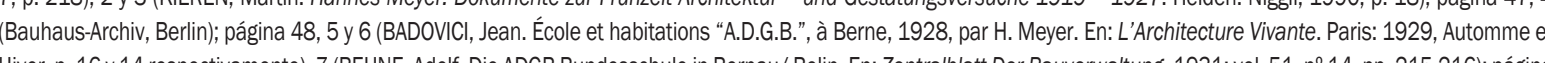

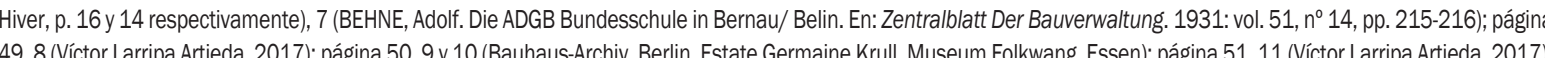

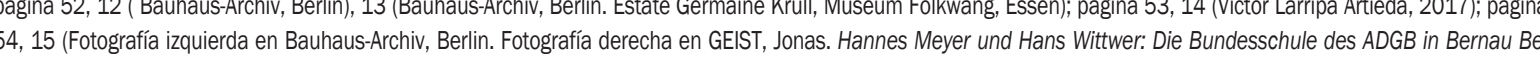
Berlin: 1930-1983. Potsdam: Potsdamer Verlags Buchnandlung, 1993, p. 16); página 59, 1 (Elaboración propia a partir de difierentes fuentes. Dibujo realizado por Roberto

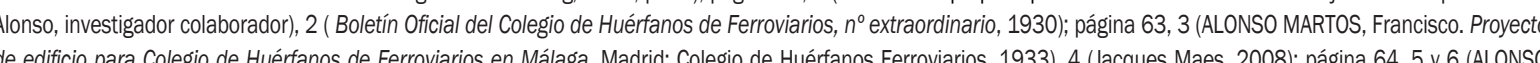

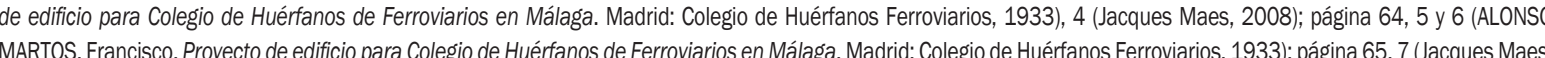

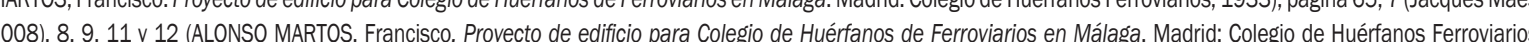
1933), 10 (Mar Loren-Méndez, 2016), 11 (ALLONSO MARTOS, Francisco. Proyecto de edificio para Colegio de Huếranos de Ferroviarios en Málaga. Madrid: Colegio de

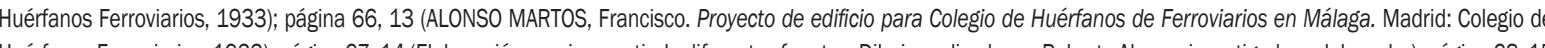

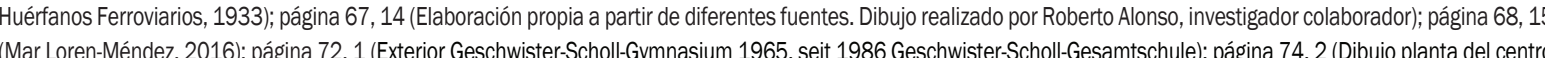

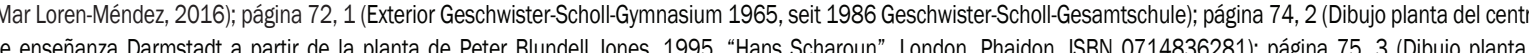
xonometría de aula para el grupo inferior, grupo intermedio y el grupo superior para el provecto del colegio en Darmstadt a partir de hitp hlescolano.blosspotcon

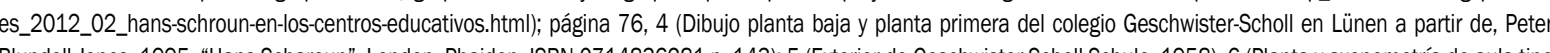
Blundell Jones, 1995, "Hans Scharoun", London, Pharidon. ISBN 0714836281.p. 143); 5 (Exterior de Geschwister-Scholl.Schule, 1958), G 6 Plantay axonometría de aula tipo en el colegio Geschwister-Scholl en Lünen a partir de, Peter Blundell Jones, 1995, "Hans Scharoun", London, Phaidon. ISBN 0714836281 p. 142), 7 Vista interior aula

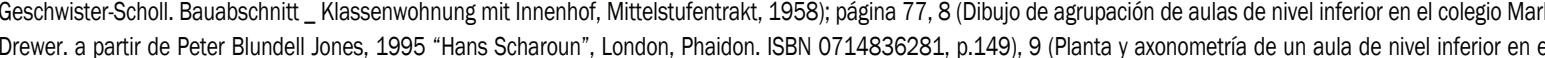
colegio Marl-Drewer. a partir de Peter Blundell Jones, 1995 "Hans Scharoun", London, Phaidon. ISBN 0714836281, p.149); página 78, 10 (Teatro del colegio Geschwister.

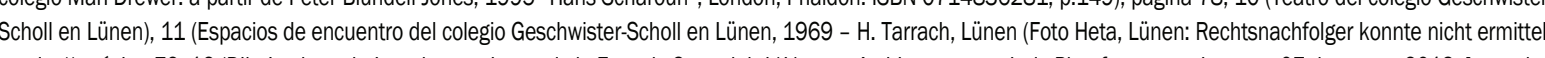

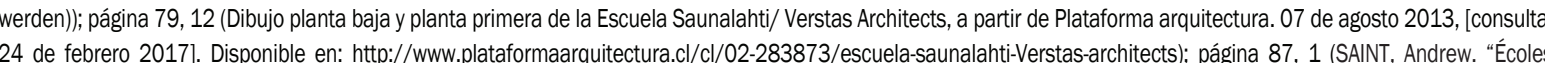

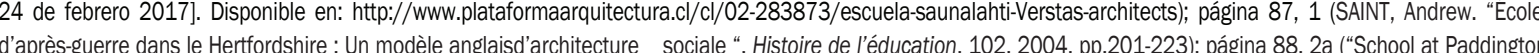

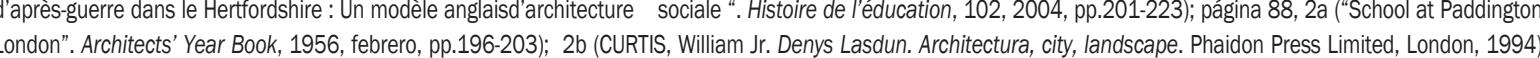

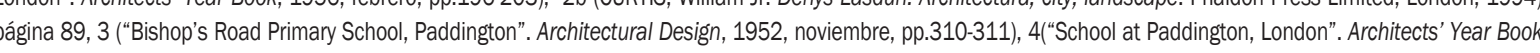
1956, febrero, pp.196-203), 5a "'School at Paddington, London". Architects's Year Book. 1956, feberero, p.196-203), 5b ""Bishop's Road Primary School, Paddington"

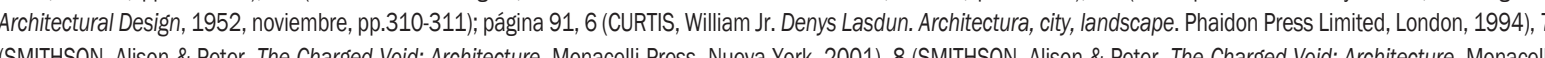

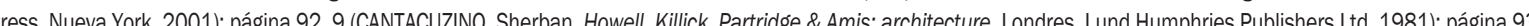

10 (CANTACUZINO, Sherban. Howell, Killick, Partridge \& Amis: architecture. Londres, Lund Humphries Publishers Ltd, 1981), 11 "PPimlico Comprehensive". Architectura

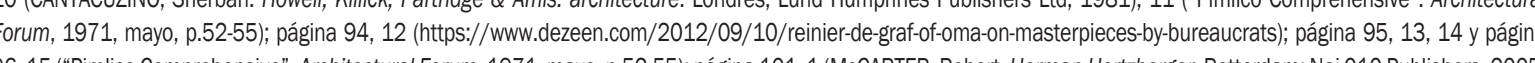
96, 15 ("Pimlico Comprenensive". Architectural Forum, 1971, mayo, p.52-55); página 101, 1 (McCARTER, Robert. Herman Hertzerger. Rotterdam: Nai 10 Publishers, 2005 ,

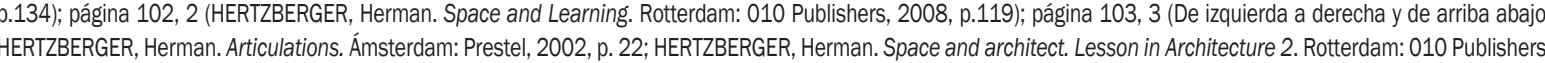

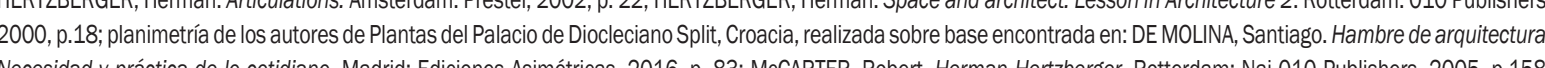
政

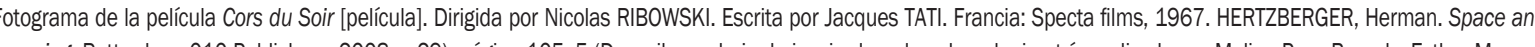

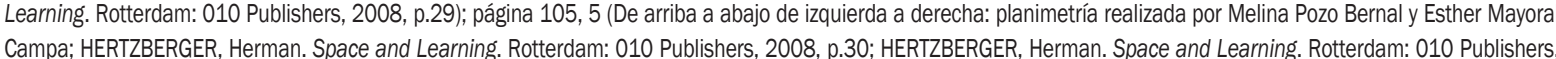

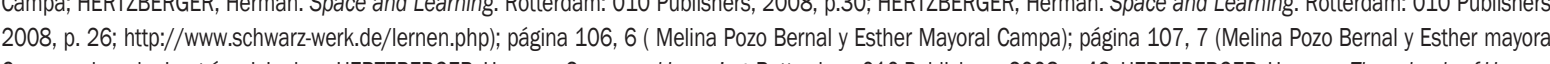

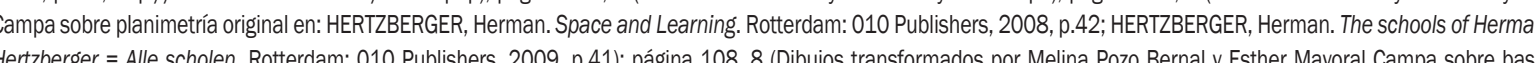
original en: HERTZZERGER, Herman. Space and Learming. Rotterdam: 010 Publishers, 2008, p. 83);página 109, 9 (Melina Pozo Bernal y Esther Mayoral Campa sobre planimetría existente en: MCCARTER, Robert. Herman Hertzberger. Rotterdam: Nai 1010 Publishers, 2005, p. 144 Y HERTZZERGGER, Herman. The schools of Herman Hertzberger

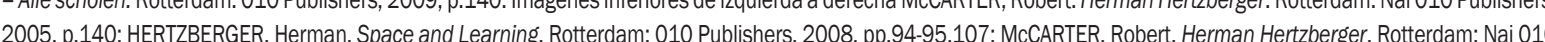

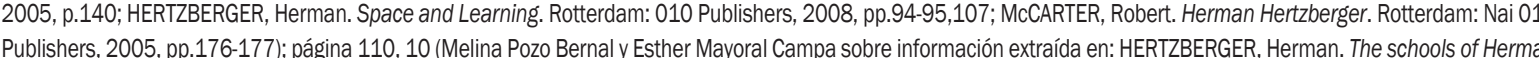
Hertzberger = Alle scholen. Rotterdam: 010 Publishers, 2009, pp.130,118. Fotograffias de arriba a abajo McCARTER, Robert. Herman Hertzberger. Rotterdam: Nai 010 Publishers, 2005, pp.184, 188, 185); página 111, 11 (Melina Pozo Bernaly Esther Mayoral Campa sobre información extraída en: McCARTER, Robert. Herman Hertzberger. Roterdam. Warto

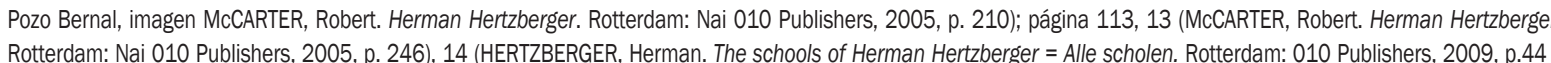
Mextraída en:

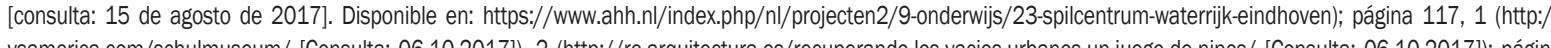

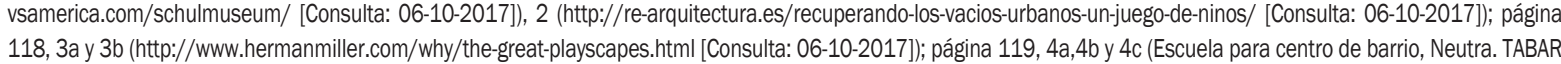

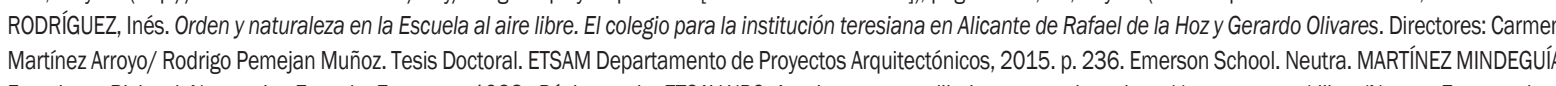

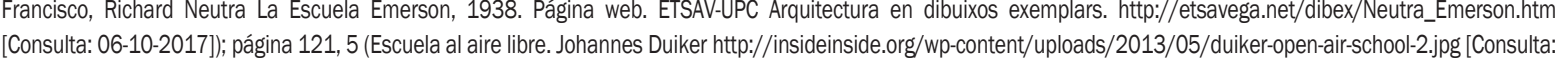

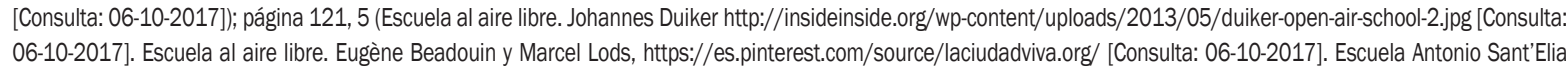

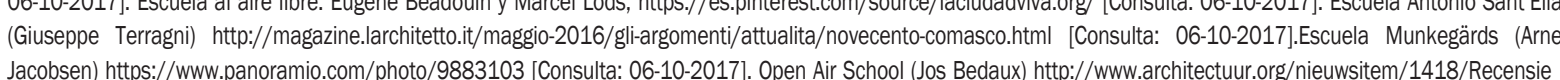

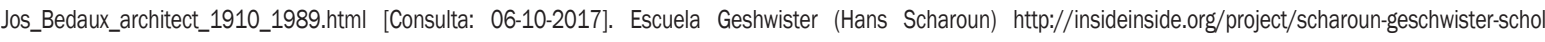

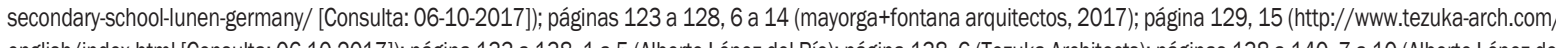

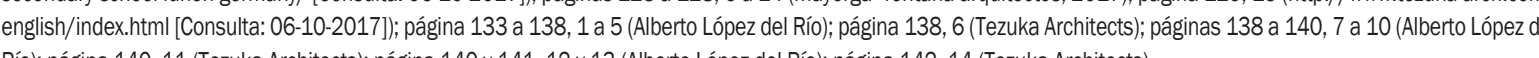

